ARTICLE

\title{
IL-21 and IFN $\alpha$ therapy rescues terminally differentiated NK cells and limits SIV reservoir in ART-treated macaques
}

\author{
Justin Harper (1) 1,11, Nicolas Huot (D) 2,11, Luca Micci ${ }^{1}$, Gregory Tharp (D) ${ }^{3}$, Colin King ${ }^{1}$, Philippe Rascle ${ }^{2,4}$, \\ Neeta Shenvi ${ }^{5}$, Hong Wang ${ }^{1}$, Cristin Galardi 6,7, Amit A. Upadhyay ${ }^{3}$, Francois Villinger ${ }^{8}$, Jeffrey Lifson ${ }^{9}$, \\ Guido Silvestri, ${ }^{1} 10$, Kirk Easley (1) ${ }^{5}$, Beatrice Jacquelin (1) ${ }^{2}$, Steven Bosinger (1) 1,3,10, \\ Michaela Müller-Trutwin (10) ${ }^{2,11}$ \& Mirko Paiardini (i] 1,10,11网
}

Unlike HIV infection, which progresses to AIDS absent suppressive anti-retroviral therapy, nonpathogenic infections in natural hosts, such African green monkeys, are characterized by a lack of gut microbial translocation and robust secondary lymphoid natural killer cell responses resulting in an absence of chronic inflammation and limited SIV dissemination in lymph node Bcell follicles. Here we report, using the pathogenic model of antiretroviral therapy-treated, SIVinfected rhesus macaques that sequential interleukin-21 and interferon alpha therapy generate terminally differentiated blood natural killer cells $\left(\mathrm{NKG} 2 \mathrm{a} / \mathrm{c}^{\text {low }} \mathrm{CD} 16^{+}\right)$with potent human leukocyte antigen-E-restricted activity in response to SIV envelope peptides. This is in contrast to control macaques, where less differentiated, interferon gamma-producing natural killer cells predominate. The frequency and activity of terminally differentiated $\mathrm{NKG} 2 \mathrm{a} / \mathrm{c}^{\mathrm{low}} \mathrm{CD} 16^{+}$natural killer cells correlates with a reduction of replication-competent SIV in lymph node during antiretroviral therapy and time to viral rebound following analytical treatment interruption. These data demonstrate that African green monkey-like natural killer cell differentiation profiles can be rescued in rhesus macaques to promote viral clearance in tissues.

\footnotetext{
${ }^{1}$ Division of Microbiology and Immunology, Yerkes National Primate Research Center, Emory University, Atlanta, GA, USA. ${ }^{2}$ Institut Pasteur, Unité HIV, Inflammation et Persistance, Paris, France. ${ }^{3}$ Nonhuman Primate Genomics Core, Yerkes National Primate Research Center, Emory University, Atlanta, GA, USA. ${ }^{4}$ Université Paris Diderot, Sorbonne Paris Cité, Paris, France. ${ }^{5}$ Department of Biostatistics and Bioinformatics, Rollins School of Public Health, Emory University, Atlanta, GA, USA. ${ }^{6}$ UNC HIV Cure Center and Department of Medicine, University of North Carolina at Chapel Hill, Chapel Hill, NC, USA. ${ }^{7}$ HIV Discovery, ViiV Healthcare, Research Triangle Park, NC, USA. ${ }^{8}$ Department of Biology, New lberia Research Center, University of Louisiana at Lafayette, New Iberia, LA, USA. ${ }^{9}$ AIDS and Cancer Virus Program, Frederick National Laboratory for Cancer Research, Frederick, MD, USA. ${ }^{10}$ Department of Pathology and Laboratory Medicine, Emory University School of Medicine, Atlanta, GA, USA. ${ }^{11}$ These authors contributed equally: Justin Harper, Nicolas Huot, Michaela Müller-Trutwin, Mirko Paiardini. ${ }^{凶}$ email: mirko.paiardini@emory.edu
} 
$\mathrm{N}$ atural killer (NK) cells are "licensed" with functional competence following education with self-major histocompatibility complex (MHC) class I molecules ${ }^{1}$. In particular human leukocyte antigen (HLA)-E, the ligand for the inhibitory CD94/NKG2a receptor ${ }^{2-4}$, is positively regulated by HLA-A expression and inhibits NK cell-mediated lysis ${ }^{5,6}$. In a companion manuscript ${ }^{7}$, Huot et al. define NK cell differentiation states based on their education via NKG2a and expression of CD16 (Fc $\gamma$ RIII), an activating $F_{c}$ receptor that mediates antibody-dependent cell-mediated cytotoxicity $(\mathrm{ADCC})^{8}$, and demonstrate that nonpathogenic SIVagm infection in African green monkeys (AGMs) imprints the maturation of NK cells inducing terminally differentiated $\mathrm{NKG} 2 \mathrm{a}^{\text {low }} \mathrm{CD} 16^{+} \mathrm{NK}$ cells, which express high levels of interleukin (IL)-21R. It has been previously demonstrated that NK cell functionality is responsive to immunotherapies with IL-21 and interferon alpha (IFNa). For example, ex vivo IL-21 treatment expanded CD16 ${ }^{+} \mathrm{NK}$ cells ${ }^{9}$, antagonized the IL-15-dependent expansion of resting NK cells ${ }^{10}$, and reverses hypo-responsiveness via the STAT1 and PI3K-AKTFOXO1 pathways ${ }^{11}$. Likewise, ex vivo IFNa therapy upregulates IL-15-mediated NK cell cytotoxicity ${ }^{12}$, including CD107a degranulation and ADCC activity ${ }^{13,14}$, and downregulates IL-21R expression $^{15}$; furthermore, in vivo IFNa-induced NK cell cytotoxicity correlates with reductions in HIV-DNA during antiretroviral therapy $(\mathrm{ART})^{16}$. In SIVagm infection, systemic IL-15 was associated with NK cell proliferation in lymph node (LN), while systemic IFNa correlated with NK cell cytotoxicity in $\mathrm{LN}^{17}$. Given previous findings on a role of IL-21 and IFNa in regulating $\mathrm{NK}$ cell function, and that $\mathrm{NKG} 2 \mathrm{a}^{\text {low }} \mathrm{CD} 16^{+} \mathrm{NK}$ cells are generated while expressing high levels of IL-21R in nonpathogenic SIV infection, we sought to determine whether immunotherapy with IL-21 and IFNa rescues AGM-like profiles of NK cell maturation and activity in SIV-infected rhesus macaques (RMs).

\section{Results}

IL-21 and rIFNa immunotherapies are biologically active in SIV-infected, ART-treated RMs. Sixteen RMs were intravenously (i.v.) infected with $\mathrm{SIVmac}_{239}$ and at day (d) 35 postinfection (p.i.) initiated triple formulation $\mathrm{ART}^{18}$, which was maintained for 13 months (Fig. 1a and Supplementary Table 1). Prior to ART initiation, the RMs RPk11 and RNa12 mimicked pre-established virologic and immunologic features of controllers; hence, both were not assigned to an experimental group, but excluded from analyses and followed as a part of a study aimed at characterizing post-treatment viral control. Among the remaining 14 RMs, 9 were administered rhesus rIL-21-IgFc (IL-21) at $\mathrm{d} 42$ and d189 p.i. in two cycles of four doses given once per week followed by weekly rhesus IFNa-IgFc (rIFNa) starting at d323 (3 doses) and d383 p.i. (2 doses; i.e., ART + IL- $21+$ rIFNa, cytokine-treated). The cytokine-treated RM 172_10 was euthanized at d66 p.i. due to AIDS-defining conditions. Five RMs served as cytokine treatment-naive, ART-only controls (i.e., controls). ART was withdrawn at $\mathrm{d} 402$ p.i. and, given attenuation in IFN signaling upon sustained therapy ${ }^{19}$, cytokine treatment-experienced RMs were transitioned to human PEGylated-IFNa (PEG-IFNa; 7 doses, once every 6-8 days, subcutaneous (s.c.), $7 \mu \mathrm{g} / \mathrm{kg}$; i.e., cytokine-treated) followed by necropsy in 6 months. IL-21 and PEG-IFNa sequential therapies were well tolerated without clinical complications as anticipated based on prior monotherapy administration in SIV-infected $\mathrm{RMs}^{20,21}$. Plasma viral loads amid ART revealed no treatment-related impact on the kinetics of viral suppression or rate of viral reactivation (Fig. 1b, c).

To confirm biological activity, we sought to recapitulate observations that IL-21 attenuates residual T-cell immune activation and improves mucosal immunity during ART ${ }^{20,22}$. In cytokine-treated RMs, ART with IL-21 treatment was superior, as compared to ART-only controls, in rapidly and significantly reducing immune activation (HLA-DR ${ }^{+} \mathrm{CD} 38^{+}$) in memory $\mathrm{CD} 4^{+} \mathrm{T}$-cells from peripheral blood mononuclear cells (PBMCs; Fig. 1d with representative stains and gating strategy in Fig. 1e and Supplementary Fig. 1a, respectively). A similar early reduction following IL-21 treatment was found in rectal biopsy (RB) for the levels of immune activation in memory CD4 ${ }^{+}$T-cells (Supplementary Fig. 2a) and proliferation $\left(\mathrm{Ki}-67^{+}\right.$) in $\mathrm{CD}^{+}{ }^{-} \mathrm{T}$-cells (Supplementary Fig. 2j); however, changes in RB did not sustain for long term and treatment did not substantially impact activation or proliferation in $\mathrm{CD}^{+}$and $\mathrm{CD}^{+}$T-cells from LN (Supplementary Fig. 2). In addition, IL-21 therapy significantly enhanced Th17/Th22 functionality based on the expression of IL-2 and TNF- $\alpha$ (Supplementary Fig. 3). The efficacy of rIFNa amid long-term ART was confirmed by the upregulation of IFN-stimulated genes (ISGs) in PBMCs at $2 \mathrm{~h}$ post-treatment relative to control RMs (Fig. 1f), which utilized six rIFNa-treated and two of five control RMs that were not described in this manuscript. In a different historical cohort ${ }^{23}$, we also confirmed that ISGs are induced by pathogenic SIVmac infection and are significantly reduced, but not fully normalized, by ART (Supplementary Fig. 4), as is observed in natural hosts ${ }^{24,25}$. Cytokine treatment reduced the frequency of HLA-E ${ }^{+} \mathrm{CD}^{+}{ }^{+} \mathrm{T}$-cells (Fig. 1g, representative gating strategy in Supplementary Fig. 1b); however, it did not impact the frequency of $\mathrm{NKG} 2 \mathrm{a} / \mathrm{c}^{+} \mathrm{CD}^{+}$T-cells (Fig. 1h, representative gating strategy in Supplementary Fig. 1c) nor did it enhance T-cell responses whether by T-bet expression, which regulates Th1 cytokine expression ${ }^{26}$, or by IFN- $\gamma$ ELISpot following stimulation with SIV-Gag or -envelope (Env) peptides (Supplementary Fig. 5; T-bet gating strategy given in Supplementary Fig. 1d). We then analyzed if the cytokine therapy enhanced NK cell activity by exposing them to MHC-I-deficient target cells with induced HLA-E loaded with SIVmac $_{239 / 251}$ Env peptides. By measuring levels of surface CD107a expression, we calculated the SIV-Env-specific, HLA-E-restricted NK cell activity (raw data of CD107a expression by co-culture condition are given in Supplementary Fig. 6-c; representative stains shown in Supplementary Fig. 6d-f; see Eq. 1). IL-21 administration led to a significant retention, which was sustained following rIFNa administration, of the Env-specific NK cell activity independent of viremia (Fig. 1i), indicating that the designed immunotherapy impacted NK cell imprinting while $\mathrm{CD}^{+} \mathrm{T}$-cell responses remained tepid.

Cytokine therapy reduces replication competent virus in lymphoid tissue, which is uniquely correlated with Env-specific NK cell activity. As cytokine therapy enhanced Env-specific NK cell activity, we sought to determine the impact on viral persistence amid ongoing ART. Independent of ART-mediated viral suppression, cytokine therapy failed to reduce the content of total cellassociated SIV-RNA (Fig. 2a-c) or -DNA (Fig. 2d-f) as determined by quantitative reverse transcription polymerase chain reaction (qRT-PCR) in bulk PBMCs or $\mathrm{LN}$ when compared to controls; yet, cell-associated SIV-DNA in RB was lower in cytokine-treated animals as compared to controls $(p=0.0576$ at d374 p.i.; Fig. 2f). Moreover, in cytokine-treated RMs, IL-21 therapy (d217 p.i.) significantly decreased the frequency of LN $\mathrm{CD}^{+}$cells harboring replication competent virus relative to controls, as determined by quantitative viral outgrowth assay (QVOA) (Fig. 2g). In three out of four controls, viral suppression due to ongoing ART (d217 to $\mathrm{d} 374$ p.i.) resulted in a nonsignificant reduction of replication competent virus. In cytokinetreated RMs, subsequent rIFNa therapy did not further decline the replication competent viral content, which remained significantly lower than controls also at d374 p.i. (Fig. 2g). Consistent with previous observations ${ }^{20}$, the IL-21 impact on replication competent virus was likely unrelated to SIV-specific T-cell responses 
a

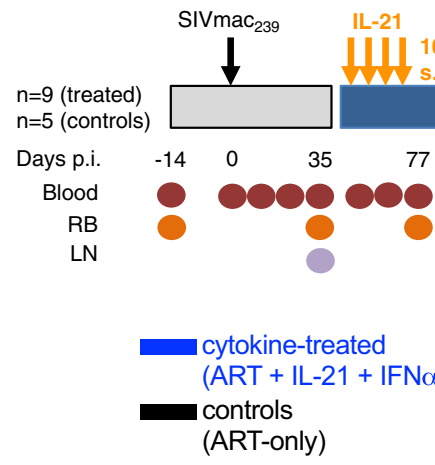

b

SIV-RNA

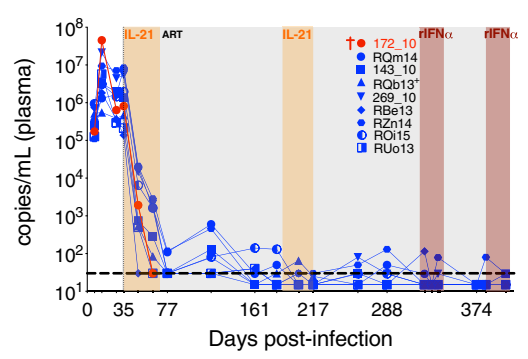

c
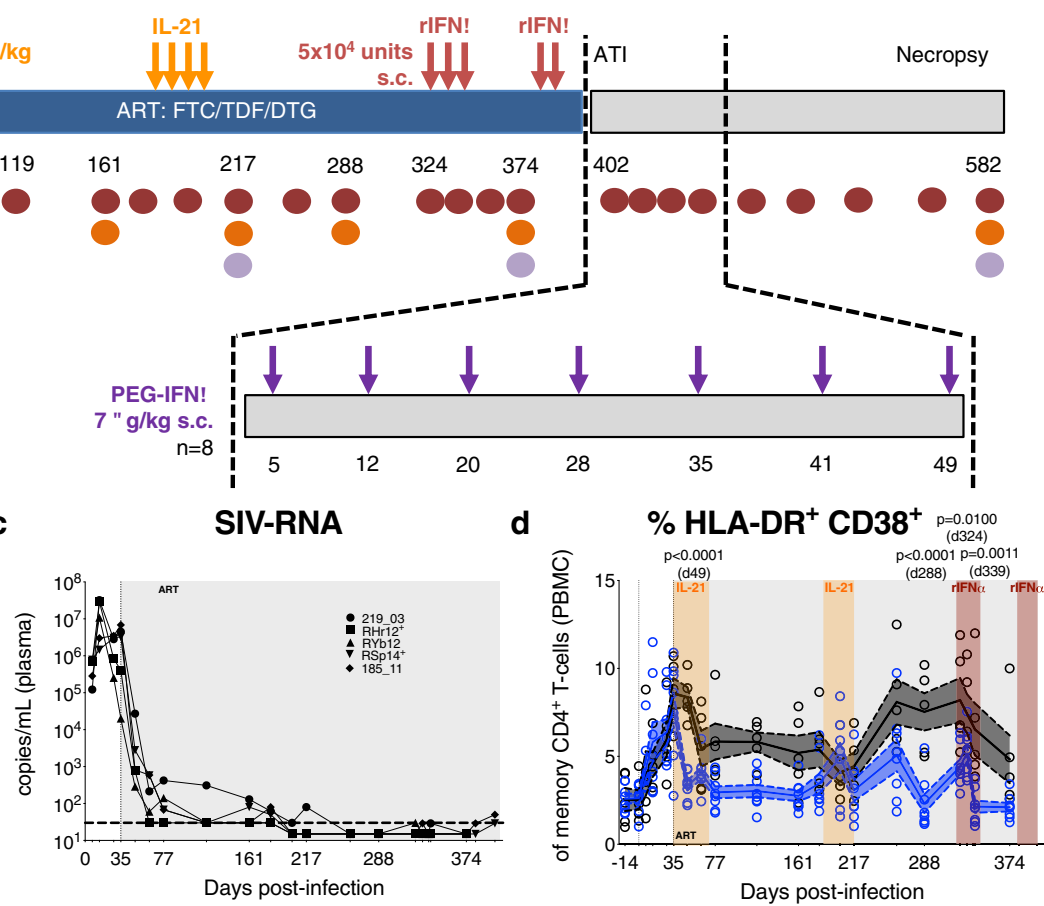

e

of memory CD4+ ${ }^{+}$-cells (PBMC)

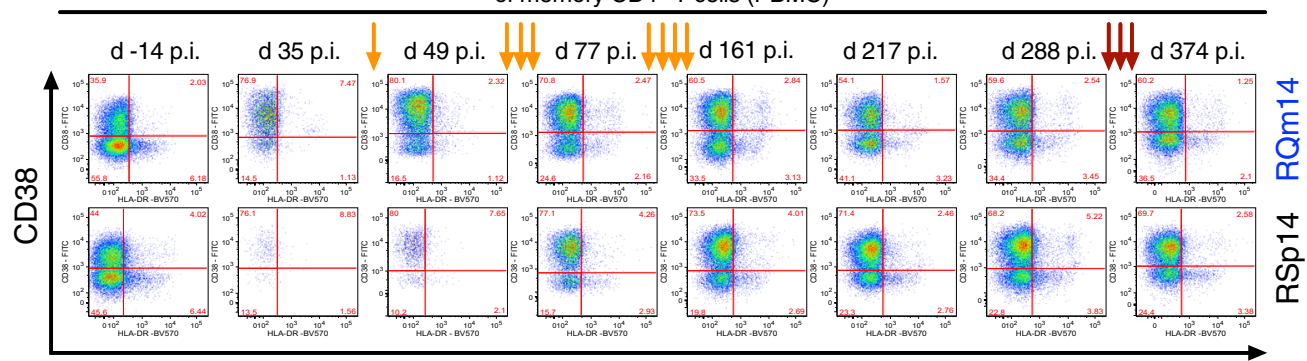

HLA-DR

$\log _{2}$ fold-change ISG expression (PBMC)

f

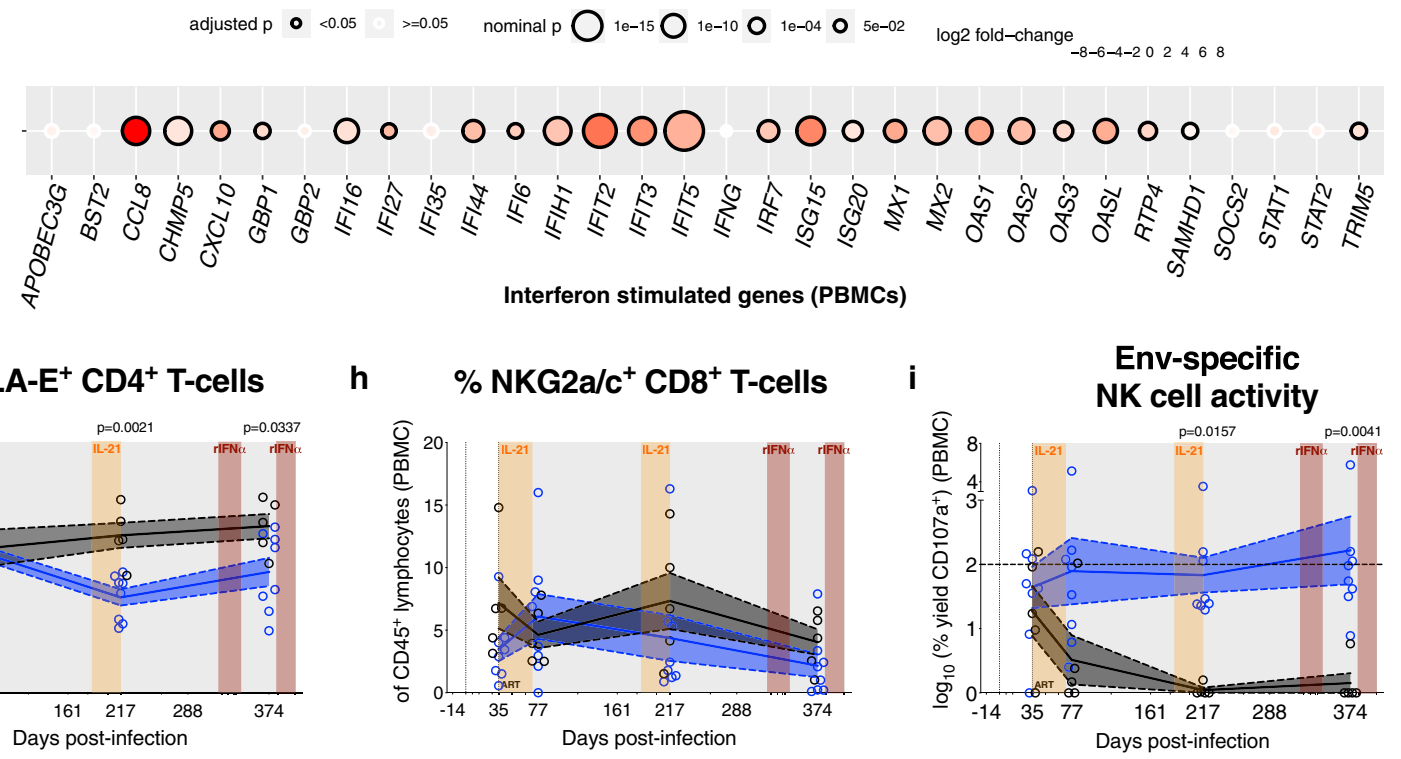

(Supplementary Fig. 5d, e). To further understand the effect on the viral reservoir, measures of SIV reservoir content were correlated against measures of T-cell immune activation, $\mathrm{T}$-cell proliferation, HLA-E ${ }^{+} \mathrm{CD}^{+}{ }^{+}$T-cells, NKG2a/c ${ }^{+} \mathrm{CD} 8^{+}$T-cells, and Envspecific NK cell activity (Fig. 2h). The content of cell-associated SIV-RNA and -DNA, which were not impacted by therapy, positively correlated with T-cell activation and proliferation as expected ${ }^{27,28}$. The frequency of $\mathrm{LN} \mathrm{CD} 4^{+}$cells harboring replication competent virus was also positively correlated with $\mathrm{T}$-cell activation and proliferation in blood, but not in LN. Given the enrichment of effector cells in blood as compared to lymphoid tissues $^{29}$, it is plausible that the levels of T-cell activation and 
Fig. 1 IL-21 and rIFN $\alpha$ immunotherapies are biologically active in SIV-infected, ART-treated RMs. a Cartoon schematic of study design as detailed in the Results and Methods. Plasma viral loads (SIV-RNA copies/mL) were longitudinally measured by qRT-PCR with individual b cytokine-treated (ART + IL-21 + IFN $\alpha$; blue; $n=9 \mathrm{RMs}$ ) and c control (ART-only; black; $n=5 \mathrm{RMs}$ ) RMs represented by distinct shapes. The dashed horizontal line represents the assay's limit of detection (30 copies $/ \mathrm{mL}$ ) with undetectable events plotted as 15 copies $/ \mathrm{mL}$. d The frequency of immune activation (HLA-DR+ ${ }^{+} \mathrm{CD}_{38}{ }^{+}$) was quantified in memory $\left(\mathrm{CD}^{+} 5^{+}\right) \mathrm{CD} 4^{+} \mathrm{T}$-cells in PBMCs and e representative flow cytometry plots are given at critical time points (as indicated above) for a cytokine-treated (RQm14) and control RMs (RSp14; 16 of 260 single-replicate stains). f The expression of IFN-stimulated genes (ISGs) was measured by RNA-seq in PBMCs at $2 \mathrm{~h}$ post-rIFN $\alpha$ and was calculated as a $\log _{2}$ fold-change between rIFN $\alpha$-treated $(n=6)$ and control RMs $(n=5)$, which is represented as a double-gradient heatmap. The size of each data point corresponds inversely to the $\log _{10}$-transformed nominal $p$ value with significant $(p<0.05)$ adjusted $p$ values indicated by a black border. Using DESeq2, data were analyzed with a two-sided $(95 \% \mathrm{CI})$ Wald test using the Benjamini-Hochberg

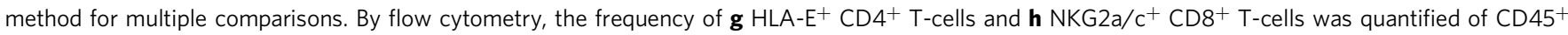
lymphocytes in PBMCs. i NK cells isolated from PBMCs were cultured alone or the presence of K562-HLA-E*0101 cells either unloaded or loaded with $\mathrm{SIVmac}_{239 / 251}$ Env peptides. The SIV-Env-specific, HLA-E-restricted activity was calculated as the $\log _{10}$-transformed percent yield of CD107a surface expression on NK cells by flow cytometry and the horizontal dashed line represents $100 \%$ activity. $\mathbf{b}$-d, $\mathbf{g}$-i Treatment phases are indicated with the following background shading: IL-21 (orange), rIFN $\alpha$ (red), and ART (gray). d, g-i Data from individual RMs (staggered open circles) are overlaid against the mean (solid line) \pm SEM (shaded region within the dashed lines): control (black; $n=5$ ) and cytokine-treated (blue; $n=8$ ). Data were analyzed with a $\mathbf{d}, \mathbf{g}-\mathbf{i}$ two-sided $(95 \% \mathrm{Cl})$, two-way ANOVA with Bonferroni's correction for multiple comparisons with cross-sectional comparisons relative to controls.

proliferation in blood may better represent therapy-induced immunologic and virologic changes, including in the content of replication competent virus, in LN and other, not accessed anatomical sites. Of note, the content of LN replication competent virus, but not cell-associated SIV-DNA in tissues, displayed a unique positive correlation with $\mathrm{HLA}-\mathrm{E}^{+} \mathrm{CD} 4^{+} \mathrm{T}$-cell levels and a negative correlation with Env-specific NK cell activity; suggesting that enhanced NK cell functionality is an important mechanism for IL-21-mediated reduction of the replication competent viral reservoirs.

Cytokine therapy promotes the maturation of NKG2a/ clow $^{\text {CD16 }}{ }^{+} \mathrm{NK}$ cells with enhanced ex vivo innate activity, which correlates with the content of lymphoid replication competent virus. To better characterize the NK cell-mediated response during ART, blood NK cells were immunophenotyped for biomarkers of homing and differentiation. Cytokine therapy resulted in a non-significant increase in the frequency of total NK cells $\left(\mathrm{CD} 45^{+} \mathrm{CD} 20^{-} \mathrm{CD} 3{ }^{-} \mathrm{NKG} 2 \mathrm{a} / \mathrm{c}^{+}\right.$; Fig. 3a) and did not impact homing to the B-cell follicle as gauged by CXCR5 expression (Fig. $3 \mathrm{~b}$ ), the expression of activating receptors (i.e., NKp30, NKp80, and NKp46), or cellular activation (HLA-DR) (Supplementary Fig. 7, representative gating strategy in Supplementary Fig. 1c). NK cells were divided into distinct differentiation stages (stages $0-3$ ) based on their expression of CD16 and NKG2a/c (Fig. 3c-f; representative gating strategy and plots in Supplementary Fig. 8) ${ }^{7}$ with the caveat that the anti-NKG2a monoclonal antibody (mAb) (clone Z199) cannot distinguish between NKG2a and NKG2c in nonhuman primates (NHPs). Cytokine therapy significantly favored the generation of the terminally differentiated $\mathrm{NKG} 2 \mathrm{a} / \mathrm{c}^{\text {low }} \mathrm{CD} 16^{+}$subset (Stage 3; Fig. 3f, g) with a concomitant loss of the intermediate NKG2a/ $\mathrm{c}^{\text {high }} \mathrm{CD} 16^{+}$subset as a proportion of NK cells (Stage 2; Fig. 3e, g). Specifically, at d 374 p.i. stage 3 NK cells constitute $46.3 \pm 7.52 \%$ of total NK cells in cytokine-treated RMs as compared to $7.59 \pm 2.24 \%$ in controls. Notably, cytokine therapy did not impact the frequency of these NK cell subsets relative to $\mathrm{CD} 45^{+}$lymphocytes (Supplementary Fig. 9a-d). These data suggest that maturation was blocked in SIVmac infection, even under ART, in favor of intermediate $\mathrm{NKG} 2 \mathrm{a} / \mathrm{c}^{\text {high }} \mathrm{CD} 16^{+} \mathrm{NK}$ cells, while cytokine therapy allowed NK cells to attain terminal differentiation.

We next analyzed the innate activity of NK cells ex vivo. In cytokine-treated RMs, we observed a transient induction of IFN- $\gamma$ expression (Fig. 3h, representative gating strategy in Supplementary Fig. 1c), but an increase in CD107a degranulation, specifically within the NKG2a/clow $C D 16^{+}$subset (Fig. 3i) ${ }^{30}$. Although other NK cell differentiation subsets displayed variable levels of ex vivo innate degranulation activity, cytokine therapy skewed the total ex vivo innate activity toward being dominated by stage 3 NK cells by d374 p.i. (Supplementary Fig. 9e-h). Indeed, at all measured experimental points (d77, d217, and $\mathrm{d} 374)$, the average \%CD107a ${ }^{+}$stage $3 \mathrm{NK}$ cells were more than seven-fold higher in cytokine-treated than control RMs. To delineate the impact of cytokine therapy-mediated NK cell differentiation, the frequency of each differentiation subset was correlated against measures of SIV persistence, and innate and Env-specific NK cell activities in all RMs (Fig. 3j). The levels of intermediate $\mathrm{NKG} 2 \mathrm{a} / \mathrm{c}^{\text {high }} \mathrm{CD} 16^{+} \mathrm{NK}$ cells (stage 2 ) were positively correlated with replication competent virus in $\mathrm{LN} \mathrm{CD} 4^{+}$cells and negatively correlated with the Env-specific NK cell activity, in line with a lack of capacity of these cells to eliminate the persistent reservoir. In sharp contrast to the less mature NK cells, the levels of stages 3 NK cells were negatively and positively correlated with replication competent virus in $\mathrm{LN} \mathrm{CD} 4^{+}$cells and the Env-specific NK cell activity, respectively (Fig. 3j). These data imply that the terminally differentiated NKG2a/clow $\mathrm{CD} 16^{+} \mathrm{NK}$ cells (stage 3 ) were responsible for the Env-specific activity and possess effector responses capable of eliminating cells specifically harboring replication competent virus during ART.

The formation and activity of $\mathrm{NKG} 2 \mathrm{a}^{\text {low }} \mathrm{CD} 16^{+} \mathrm{NK}$ cells correlate with viral recrudescence following ATI. To further analyze the functional relevance of NK cell differentiation in viral persistence, all RMs underwent ART analytical treatment interruption (ATI) with cytokine-treated RMs additionally receiving ongoing PEG-IFNa (Fig. 1a). Of note, PEG-IFNa therapy has previously been suggested as able to delay viral rebound when initiated prior to $\mathrm{ATI}^{31,32}$, whereas in SIV-infected RMs, prior IL-21 monotherapy during ART is $\operatorname{not}^{20}$. Based on longitudinal plasma viremia following ATI (Fig. 4a, b), cytokine-treated RMs exhibited a significant delay in rebound ( $>200$ copies/mL) both by survival curve analysis (Fig. 4c) and by day of rebound (average $22.1 \pm 4.27$ days versus $10.6 \pm 0.98$ days; Fig. $4 d$ ). Thus, cytokine treatment modulated the kinetics of plasma rebound, as a slope (d13-d20 ATI) and mean analysis (d13 ATI, Fig. 4e). Cytokine therapy did not however impact the peak or set-point viremia relative to controls (Supplementary Fig. 10a, b) or the content of cell-associated SIV-DNA or -RNA in PBMCs (Supplementary Fig. 10c, d). Transitioning treatment-experienced RMs (i.e., cytokine-treated RMs with prior rIFNa during ART) to PEG-IFNa led to a reset in ISG expression as of $24 \mathrm{~h}$ following the first administration (d6 ATI); however, this effect was largely lost following the fifth dose by which nearly all RMs had rebounded (d37 ATI; Fig. 5a). As with rIFNa on-ART, PEG-IFNa therapy following ATI failed to improve SIV-specific T- 
cell-associated SIV-RNA
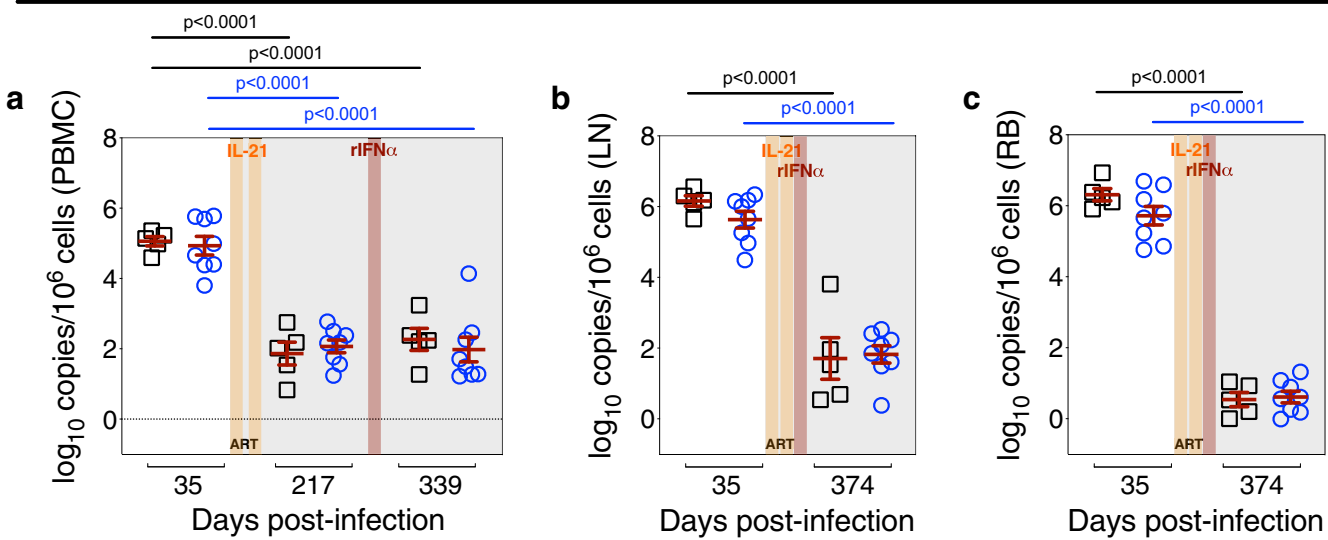

cell-associated SIV-DNA
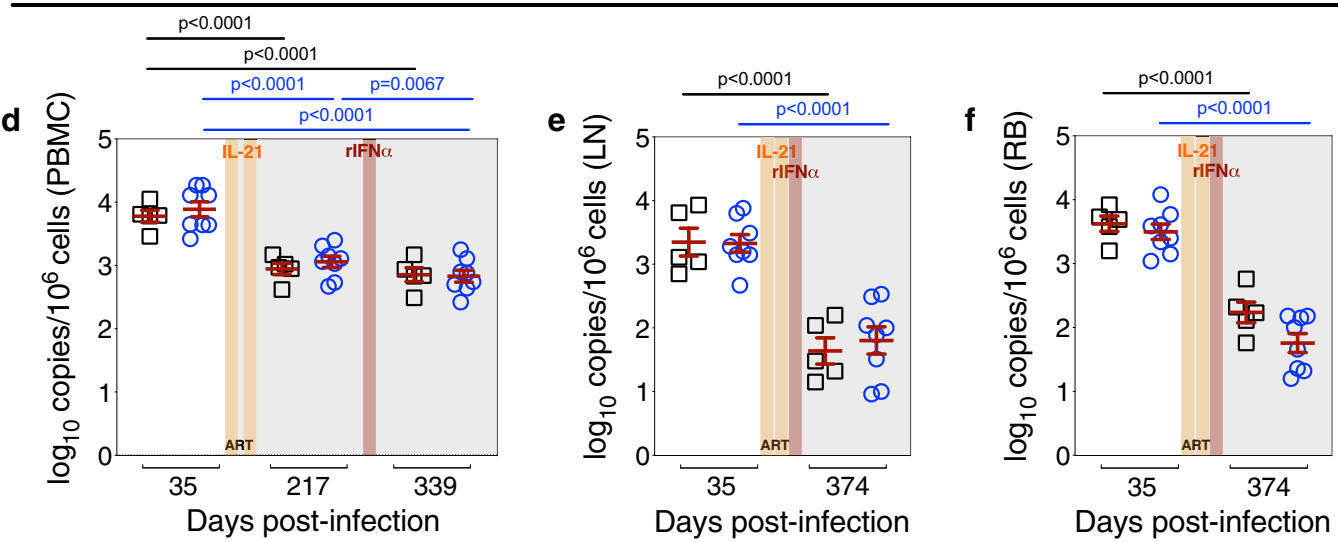

h Spearman's rank order correlation
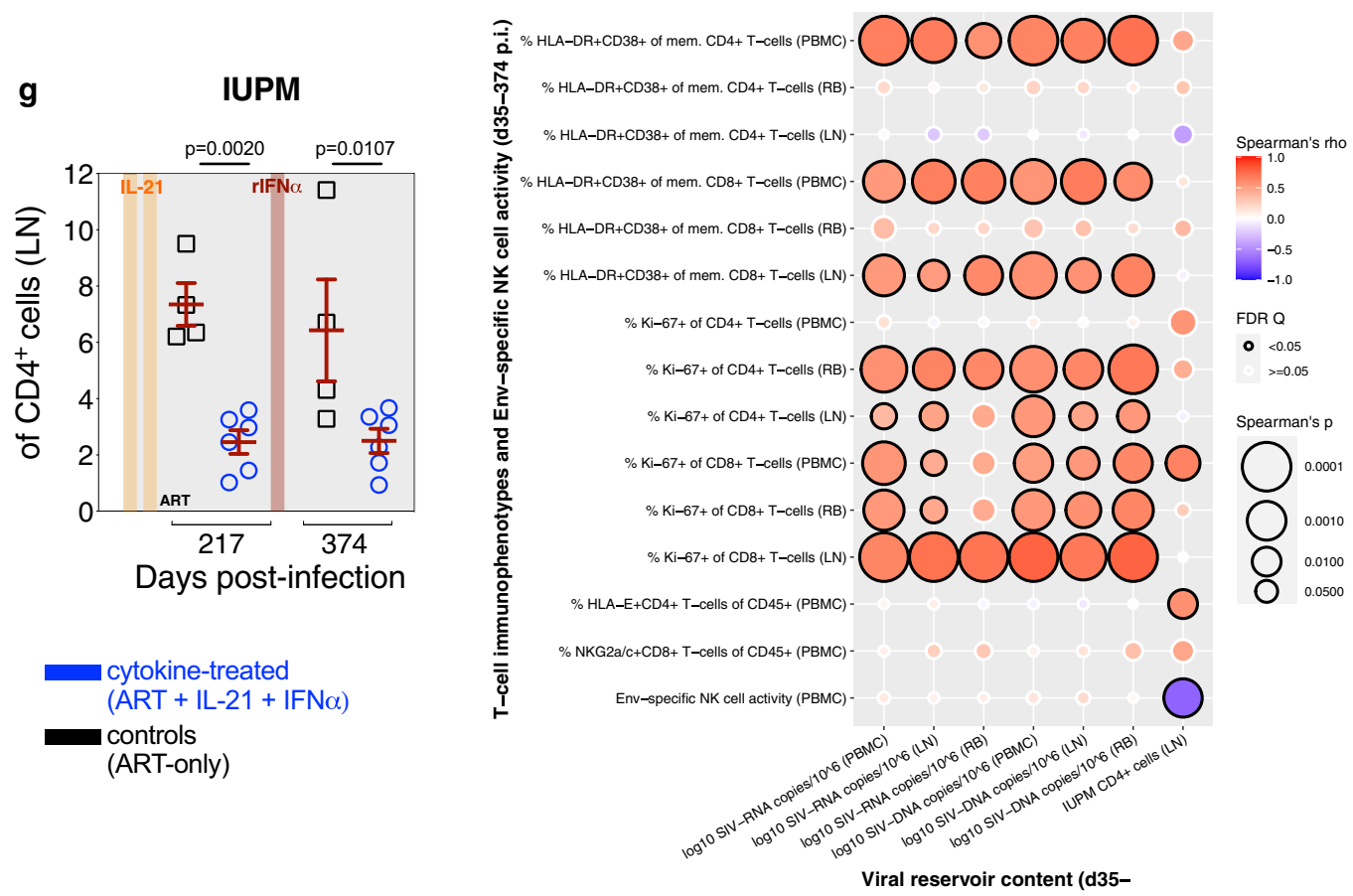

cell responses by SIV-Gag-stimulated IFN- $\gamma$ ELISpot (Supplementary Fig. 10e). At d13 post ATI, the distribution of NK cell maturation subsets in PBMCs was similar to that observed during ART with higher levels of terminally differentiated (NKG2a/ $\mathrm{c}^{\text {low }} \mathrm{CD} 16^{+}$, stage 3) NK cells with strong, innate degranulation activity (i.e., ex vivo CD107a surface expression) in the cytokine- treated animals in contrast to higher levels of the intermediate (NKG2a/chigh $\mathrm{CD} 16^{+}$, stage 2) NK cells with weak innate degranulation activity in the controls (Fig. 5b, c). In contrast, at d13 post ATI, the Env-specific activity of bulk NK cells in cytokine-treated animals was no longer statistically significantly different from controls (Fig. 5d) and tended to converge with the levels observed in 
Fig. 2 Cytokine therapy reduces replication competent virus in lymphoid tissue, which is uniquely correlated with Env-specific NK cell activity. The content of cell-associated SIV-RNA ( $\log _{10}$ copies per $10^{6}$ cells) was determined by qRT-PCR in snap frozen pellets of a PBMCs, $\mathbf{b}$ LN, and $\mathbf{c}$ RB prior to ART initiation (d35 p.i.), following two IL-21 cycles (d217 p.i.), and after one subsequent rIFNa cycle amid ongoing ART (d339 p.i. or d374 p.i.), as was cellassociated SIV-DNA ( $\log _{10}$ copies per $10^{6}$ cells) in d PBMCs, e LN, and $\mathbf{f}$ RB. a-f Data from individual RMs are overlaid against the mean \pm SEM (in red): control (ART-only, black square; $n=5 \mathrm{RMs}$ ) and cytokine-treated (ART + IL-21 + IFN $\alpha$, blue circle; $n=8$ RMs). g From cryo-preserved, magnetically isolated LN CD4 ${ }^{+}$cells, the number of infectious units per million (IUPM) cells were measured with a limiting dilution quantitative viral outgrowth assay (QVOA; 3 serial dilutions plated in triplicate) in control $(n=4)$ and cytokine-treated RMs $(n=6)$. $\mathbf{a}-\mathbf{g}$ Treatment phases are indicated with the following background shading: IL-21 (orange), rIFN $\alpha$ (red), and ART (gray). Data were analyzed with two-sided (95\%), two-way ANOVA with Bonferroni's correction with comparisons relative to controls and time. $\mathbf{h}$ The SIV reservoir contents in tissue (as indicated below) were correlated against phenotypes

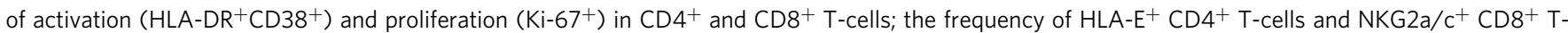
cells; and the Env-specific NK cell activity (as indicated at left) in all RMs ( $n=13$; days 35, 217, and 339/374 p.i. as matched data are available). Per each correlation the two-tailed $(95 \% \mathrm{Cl})$ Spearman's rank correlation coefficient (rho) is represented as a double-gradient heatmap and the size of each data point corresponds inversely to the $\log _{10}$-transformed Spearman's $p$ value. The false discovery rates (FDR) were calculated using SAS and significant values $(Q<0.05)$ are represented by a black border.

chronic infection prior to ART initiation (d35 p.i.) (Fig. 1i). Furthermore, at d58 post ATI, by which all RMs had experienced virologic rebound and PEG-IFNa therapy was no longer effective (Figs. $4 \mathrm{c}$ and $5 \mathrm{a}$ ), the frequency of terminally differentiated NK cells in cytokine-treated RMs converged with levels observed in controls (Supplementary Fig. 10f); indicating that during viremic conditions prior cytokine therapy alone does not lead to persistent alterations in NK cell differentiation in pathogenic models of infection. Finally, the frequency and innate activity of terminally differentiated NK cells, both on-ART (d374 p.i.) and following ATI (d13), were positively correlated with the delay in rebound of plasma viremia, whereas the frequency of intermediate NK cells was associated with poor viral control following ATI (Fig. 5e). These analyses revealed a unique and strong relationship between the cytokine therapymediated differentiation of SIV-Env-specific, HLA-E-restricted $\mathrm{NKG} 2 \mathrm{a} / \mathrm{c}^{\text {low } C D 16}{ }^{+} \mathrm{NK}$ cells and their activity during ART with the subsequent delay in viral rebound following ATI.

\section{Discussion}

Although historically underappreciated in curative approaches for chronic viral infections, these data demonstrate that HLAE-restricted NK cell responses impact SIV control in vivo, as has been found in mouse models for other viral infections $s^{33-35}$. The formation of a terminally differentiated NK cell subset $\left(\mathrm{NKG} 2 \mathrm{a} / \mathrm{c}^{\text {low }} \mathrm{CD} 16^{+}\right)$with robust innate and adaptive antiviral activities, which was found in nonpathogenic infections in $\mathrm{AGMs}^{7}$, was blocked in pathogenic SIVmac infection in favor of intermediate differentiation (NKG2a/ $/ \mathrm{c}^{\text {high }} \mathrm{CD} 16^{+}$) with heightened pro-inflammatory potential ${ }^{36}$. Notably, we have demonstrated in a pathogenic model of infection that IL-21 and IFNa treatment during ART were effective in removing this block and in promoting NK cell terminal differentiation without altering their follicular homing or inducing a de novo expansion. As terminally differentiated NK cells were correlated with reductions in lymphoid replication competent virus during ART and the delay in viral rebound after ATI, these data support a role for SIV-Env-specific, HLA-E-restricted NK cells responses in controlling SIV in tissues. These data parallel earlier observations that robust NK cell responses in natural SIV hosts are a critical determinant of viral dissemination and control in secondary lymphoid tissue ${ }^{37}$, which may represent a key factor mediating chronic inflammation and AIDS progression $^{38,39}$ in people living with HIV. It is plausible that viral control is influenced by a complex array or distinct parameters. For example, studies demonstrating CD8 ${ }^{+} \mathrm{T}$-cellmediated viral control utilized an anti-CD8 depleting mAb (MT-807R1) that would also deplete NK cells as both express the CD8a chain ${ }^{40-42}$; however, this interplay could be resolved with depletions targeting the $\operatorname{CD} 8 \beta$ chain, which is only expressed on $\mathrm{T}$ cells ${ }^{43}$. Furthermore, these models would underestimate the role of $\mathrm{NK}$ cells in viral control given our data demonstrate that $\mathrm{NK}$ cell terminal differentiation is blocked with pathogenic infection, which we postulate is a consequence of persistent inflammation and the cytokine environment in lymphoid tissue, or other factors influencing NK cell education, such as aberrant MHC expression and/or MHC peptide loading. As a caveat, although IL-21 therapy alone promoted NK cell maturation, without monotherapy control arms or further sampling we cannot determine if rIFNa treatment resulted in synergy or if NK cell responses were stably sustained between interventions, respectively; in addition, we cannot discern the role of PEG-IFNa during ATI in delaying viral rebound. Overall, these data demonstrate that AGM-like profiles of NK cell terminal differentiation, and also SIV-Env-specific, HLA-E-restricted activity, can be rescued via IL-21 and IFNa therapy in SIVmac-infected RMs, which impacts the size of the lymphoid replication competent virus during ART and viral recrudescence following ATI. As NK cell terminal differentiation was more strongly and consistently associated with viral control than the innate and HLA-Erestricted activity alone, these cells might exert additional antiviral properties, possibly including ADCC. As such, targeting NK cell differentiation states is a viable strategy when designing immunotherapy regimens to facilitate clinical HIV remission.

\section{Methods}

Study design. Sixteen female Indian-origin RMs (Macaca Mulatta) were recruited to this study and housed at YNPRC (Supplementary Table 1). All animals were Mamu$\mathrm{B}^{*} 08^{-}$and $-\mathrm{B}^{*} 17^{-}$, whereas 4 RMs were Mamu-A ${ }^{*} 01^{+}$: RHr12, RNa12, RSp14, and RQb13. RMs were deemed pathogen-free and housed as previously describe ${ }^{44}$. RMs were i.v. infected with 300 TCID50 SIVmac 239 (Fig. 1a), which was purchased from Koen Van Rompay at UC-Davis. RMs were stratified into in vivo therapy cohorts balancing for their set point plasma viral loads at day 35 p.i. and their Mamu-A*01 haplotype (Supplementary Table 1). At d35 p.i. RMs began a daily, s.c. triple formulation ART regimen consisting of tenofovir disoproxil fumarate (TDF; $5.1 \mathrm{mg} / \mathrm{kg} /$ d; Gilead Sciences), emtricitabine (FTC; $40 \mathrm{mg} / \mathrm{kg} / \mathrm{d}$; Gilead Sciences), and dolutegravir (DTG; $2.5 \mathrm{mg} / \mathrm{kg} / \mathrm{d}$; ViiV Healthcare) ${ }^{18}$ that were obtained via a material transfer agreement (MTA). Nine RMs were administered two cycles of rhesus IL-21IgFc (IL-21; 4 doses, once per week, s.c. $100 \mu \mathrm{g} / \mathrm{kg}$ ) starting at day 42 p.i. and again at day 189 p.i. All animals with prior IL-21 therapy were subsequently administered rhesus IFNa-IgFc (rIFNa; once per week, s.c. 50,000 units) starting at day 323 p.i. (3X) and day 383 p.i. (2X; i.e., ART + IL-21 + IFNa, cytokine-treated). Cytokinetreated RM 172_10 was euthanized at day 66 p.i. due to rapid progression to AIDS defining endpoints related to weight loss. Five RMs were utilized as ART-only controls (i.e., controls) and two RMs (RPk11 and RNa12) were projected to be controllers based on pre-established criteria ${ }^{45}$; hence they were excluded from analyses and not assigned to an experimental group but followed as a part of a study aimed at characterizing post-treatment control. Following ART ATI (day 402 p.i.), the eight remaining cytokine-treated RMs transitioned to human PEG-IFNa (7 doses, once every $6-8$ days, s.c. $7 \mu \mathrm{g} / \mathrm{kg}$ ) starting at day 5 post ATI. Animals were followed for 6 months following ATI and subjected to necropsy. The longitudinal characterization of ISGs via RNA-seq in PBMCs was performed in a historical cohort $(n=6$; 

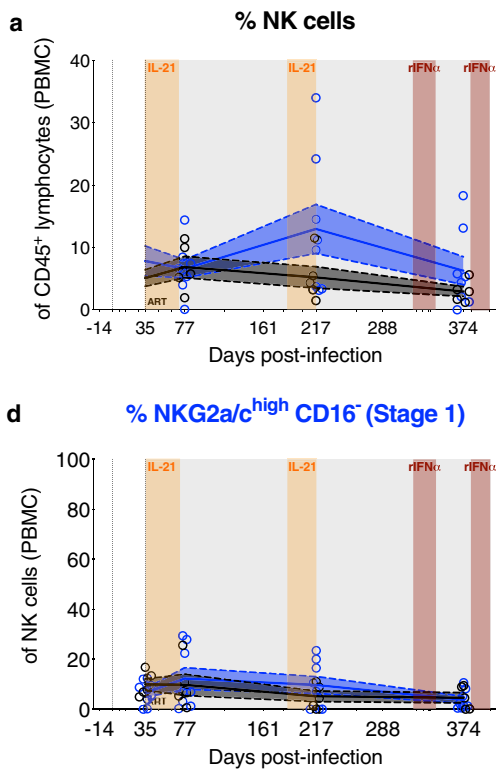

cytokine-treated $(\mathrm{ART}+\mathrm{IL}-21+\mathrm{IFN} \alpha)$ controls (ART-only)

g mean $\%$ subset of NK cells (PBMCs)
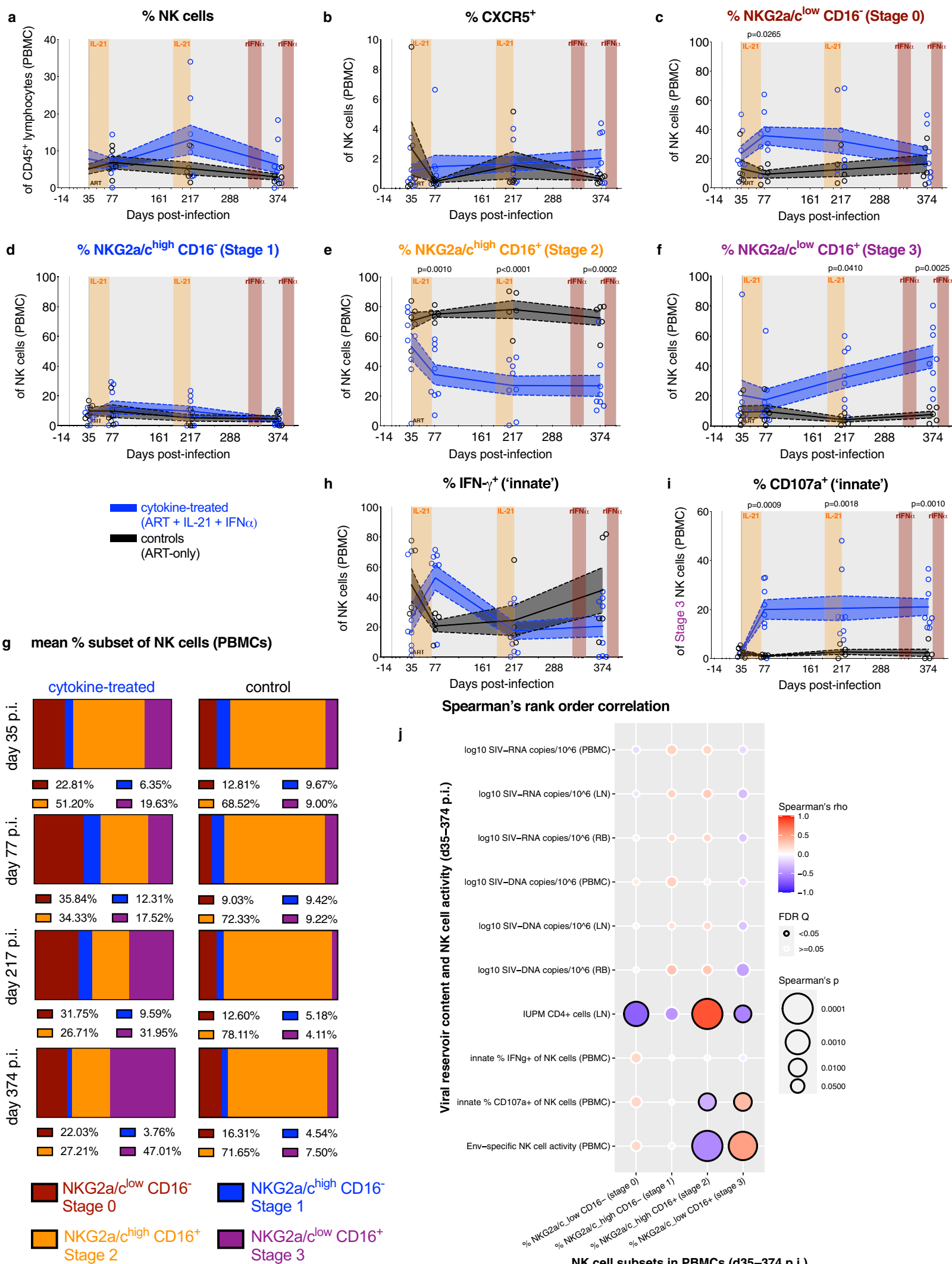

\section{Spearman's rank order correlation}

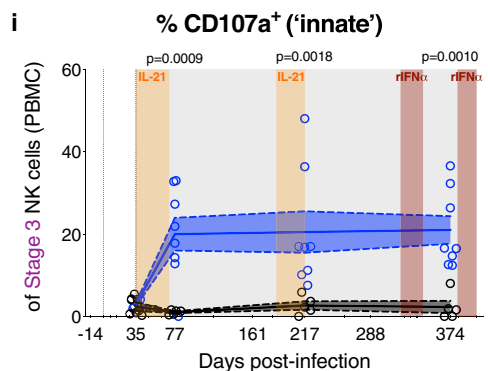

j

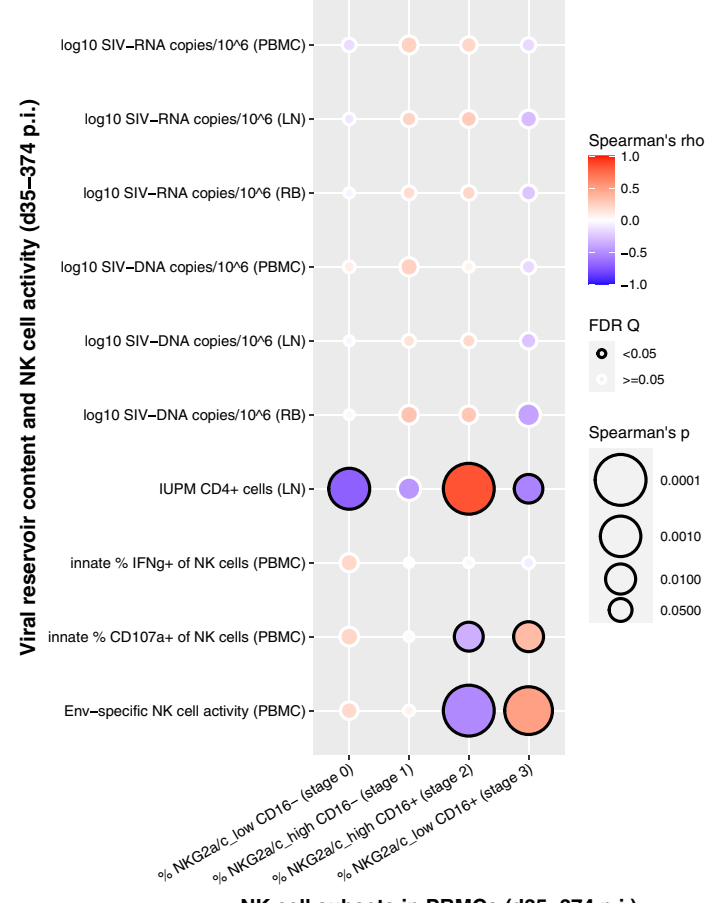

NK cell subsets in PBMCs (d35-374 p.i.)
Supplementary Fig. 4) ${ }^{23}$ in which RMs were infected i.v. with SIVmac 251 and treated with an ART regimen consisting of s.c. 3TC and TDF daily; intramuscular brecanavir weekly; and intramuscular cabotegravir once every 3 weeks.

Study approval. All animal experimentation was conducted following guidelines set forth by the Animal Welfare Act and by the NIH's Guide for the Care and Use of Laboratory Animals, 8th edition. All procedures were performed in accordance with institutional regulations and were approved by Emory University's Institutional Animal Care and Use Committee (permit 3000434). Animal care facilities are accredited by the US Department of Agriculture and the Association for Assessment and Accreditation of Laboratory Animal Care International. Proper steps were taken to minimize animal suffering and all procedures were conducted under anesthesia with follow-up pain management as needed. 
Fig. 3 Cytokine therapy promotes the maturation of NKG2a $/ c^{\text {low }}$ CD16 ${ }^{+}$NK cells with enhanced ex vivo innate activity, which correlates with the content of lymphoid replication competent virus. a The frequency of NK cells (CD45 ${ }^{+} \mathrm{CD} 2 \mathrm{O}^{-} \mathrm{CD}{ }^{-} \mathrm{NKG} 2 \mathrm{a} / \mathrm{c}^{+}$) of PBMC CD45 ${ }^{+}$lymphocytes was longitudinally measured by flow cytometry, $\mathbf{b}$ as was their CXCR5 expression. The frequency of each differentiation stage of NK cells was determined based on the following definition: c Stage 0 (red, NKG2a/clow $C D 16-$ ), d Stage 1 (blue, NKG2a/ $\mathrm{c}^{\text {high }} \mathrm{CD16}$ ), e Stage 2 (orange, NKG2a/ $\mathrm{c}^{\text {high }} \mathrm{CD16}$ ), and $\mathbf{f}$ Stage 3 (purple, NKG2a/ $\mathrm{c}^{\text {low }} \mathrm{CD}^{+} 6^{+}$). $\mathbf{g}$ The mean frequency of each NK cell differentiation stage from above was also re-visualized as a color-coded (as annotated below), parts-of-whole stacked bar plot for the cytokine-treated ( $n=8$; at left) and control RMs $(n=5$; at right) over time (indicated at left). Flow cytometry was used to quantify the ex vivo innate frequency of $\mathbf{h}$ IFN- $\gamma^{+}$(intracellular) NK cells and $\mathbf{i}$ CD107a ${ }^{+}$(surface) stage 3 (NKG2a/clow CD16 ${ }^{+}$) NK cells. a-i Data from individual RMs (staggered open circles) are overlaid against the mean (solid line) \pm SEM (shaded region within the dashed lines): control (ART-only, black; $n=5$ ) and cytokine-treated (ART + IL-21 + IFN $\alpha$, blue; $n=8$ ). Treatment phases are indicated with the following background shading: IL-21 (orange), rIFN $\alpha$ (red), and ART (gray). Data were analyzed with two-sided ( $95 \% \mathrm{Cl}$ ), two-way ANOVA with Bonferroni's correction with cross-sectional comparisons relative to controls. $\mathbf{j}$ The frequencies of each differentiation stage of NK cells in PBMCs (as indicated below) were correlated against levels of cell-associated and replication competent SIV content in tissue, and the ex vivo innate and Env-specific NK cell activities in PBMCs (as indicated at left) in all RMs ( $n=13$; days 35, 77,217 , and 374 p.i. as matched data are available). Per each correlation the two-tailed ( $95 \% \mathrm{Cl}$ ) Spearman's rank correlation coefficient (rho) is represented as a double-gradient heatmap and the size of each data point corresponds inversely to the $\log _{10}$-transformed Spearman's $p$ value. The false discovery rates (FDR) were calculated using SAS and significant values $(Q<0.05)$ are represented by a black border.

SIV-RNA

a

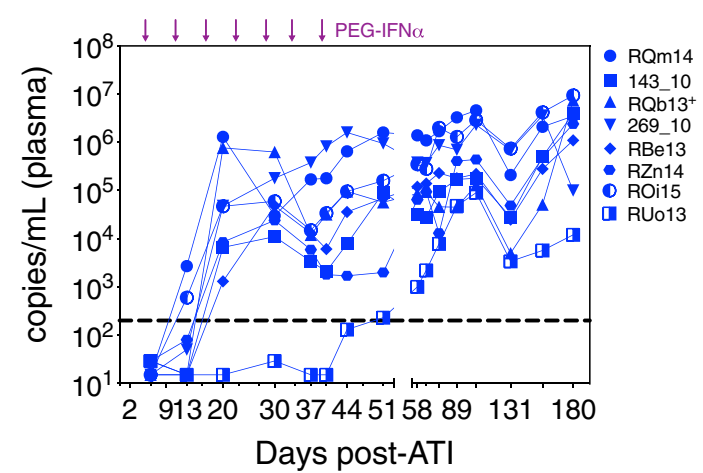

c

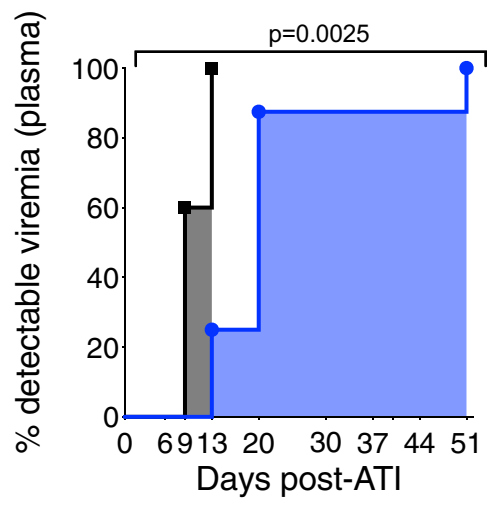

b

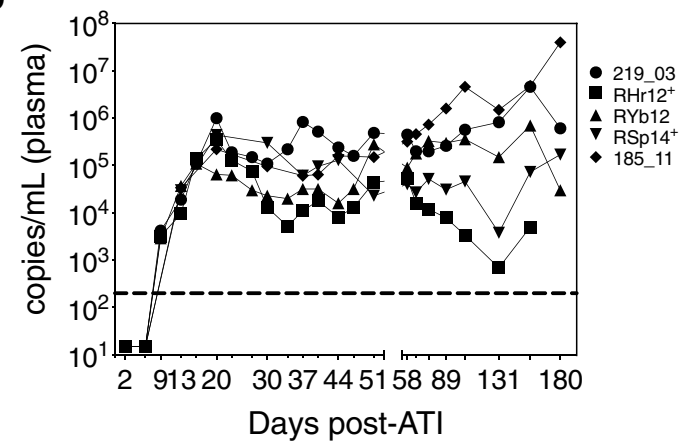

d Rebound Delay

e

SIV-RNA

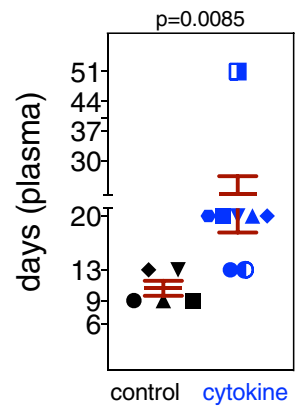

Treatment

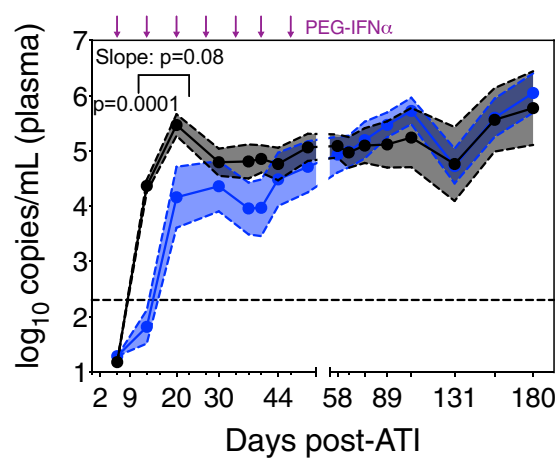

Fig. 4 Cytokine therapy delays the rebound of plasma viremia following ATI. Following ART analytical treatment interruption (ATI), the plasma SIV-RNA copies/mL were measured by qRT-PCR in each a cytokine-treated (PEG-IFN $\alpha$ with prior ART + IL-21 + rIFN $\alpha$, blue; $n=8$ RMs) and $\mathbf{b}$ control RMs (prior ART-only, black; $n=5$ RMs). The horizontal dashed line (200 copies $/ \mathrm{mL}$ ) represents the threshold for virologic rebound and PEG-IFN $\alpha$ treatments are indicated by the purple arrows above. These kinetics of plasma viremia following ATI were then re-visualized as follows: c The delay in rebound of plasma viremia was represented as a treatment-stratified survival curve, which was analyzed with a Log-rank Mantel-Cox test. $\mathbf{d}$ The delay in viral rebound, in days, per each RM was represented as a color and shape-coded symbol overlaid against the mean \pm SEM (red), which was analyzed with a two-sided (95\% CI) Mann-Whitney $U$ test. e The $\log _{10}$ SIV-RNA copies/mL are given as a longitudinal mean (solid line with closed circles) \pm SEM (color-coded shaded region within the dashed lines), which was analyzed with a two-sided $(95 \% \mathrm{CI}$ ), two-way ANOVA with Bonferroni's correction for multiple comparisons across treatments ( $\mathrm{d} 13 \mathrm{p}=0.0001$ ), and a mixed-effects linear model was used to analyze the slope between $\mathrm{d} 13$ and $\mathrm{d} 20$ post ATI (as indicated by the bracket; $p=0.08)$.

Tissue collection and processing. Collections of peripheral blood (PB), RB punches, and LN biopsies were conducted longitudinally and upon necropsy (Fig. 1a) as previously described ${ }^{44}$. EDTA PB was used for complete blood counts, and plasma was separated by centrifugation within $1 \mathrm{~h}$ of phlebotomy. PBMCs were isolated from PB by density gradient centrifugation (Ficoll-Paque Premium, GE Healthcare). RB punches were obtained by inserting an anoscope a short distance into the rectum and 20 punches were collected using a biopsy forcep. To obtain gut-derived lymphocytes, $\mathrm{RB}$ punches were digested with $1 \mathrm{mg} / \mathrm{mL}$ collagenase for $2 \mathrm{~h}$ at $37^{\circ} \mathrm{C}$ with agitation, and then filtered with a $100-\mu \mathrm{m}$ strainer to remove residual tissue fragments. For LN biopsies, the skin over the axillary or inguinal region was clipped and surgically prepped. An incision was then made in the skin and the LN was exposed by blunt dissection and excised over clamps. LNs 

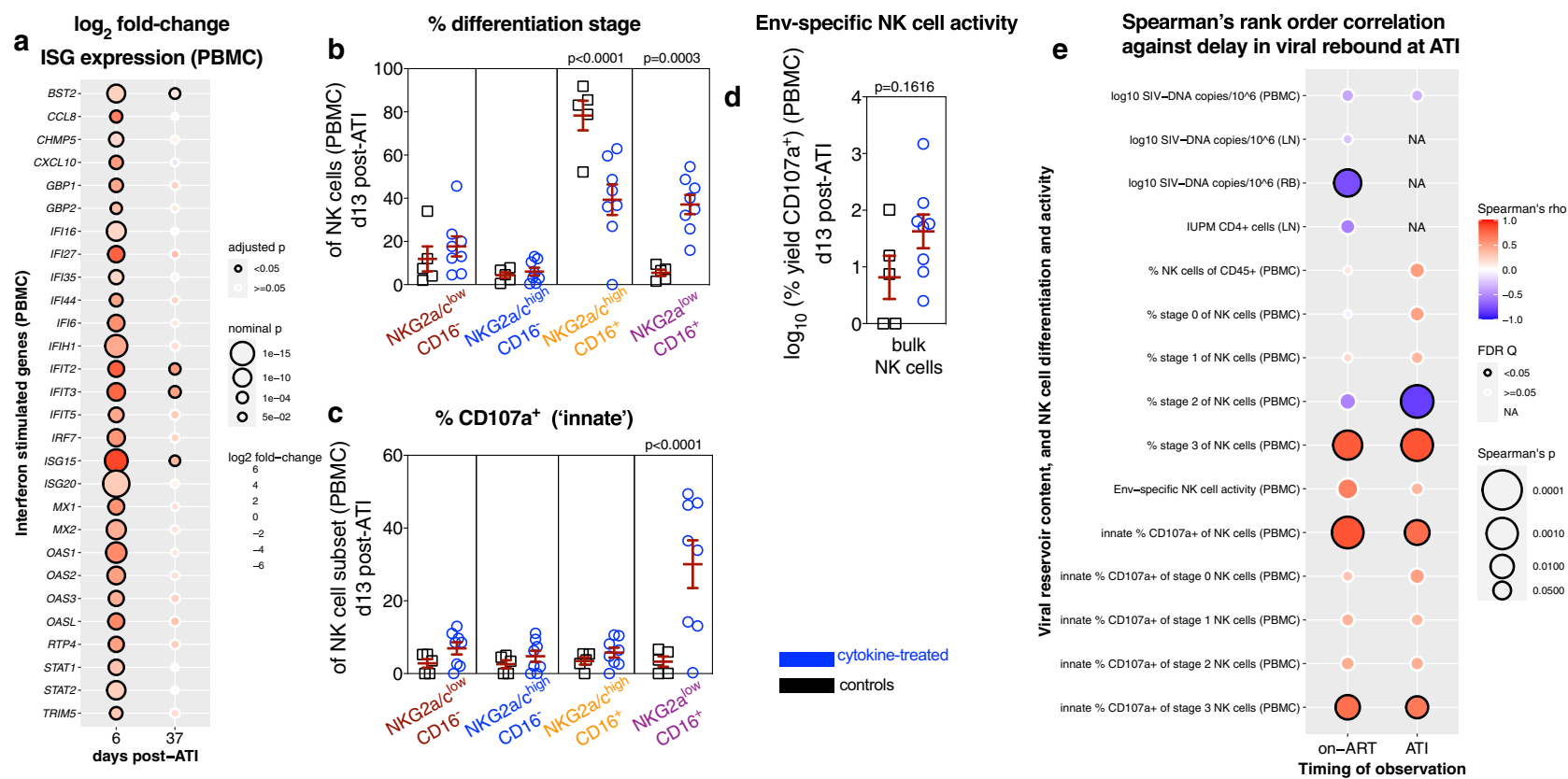

Fig. 5 The formation and activity of NKG2a ${ }^{\text {low }}$ CD16 + NK cells correlate with viral recrudescence following ATI. a From PBMCs taken $24 \mathrm{~h}$ following the first ( $\mathrm{d} 6$ post $\mathrm{ATI}$ ) and the fifth PEG-IFN $\alpha$ dose (d37 post ATI), the expression of interferon-stimulated genes was calculated as a cross-sectional log ${ }_{2}$ foldchange between cytokine-treated $(n=8)$ and control $(n=5)$ RMs, which is represented as a double-gradient heatmap. The size of each data point corresponds inversely to the $\log _{10}$-transformed nominal $p$ value with significant $(p<0.05)$ adjusted $p$ values indicated by a black border. Using DESeq2, data were analyzed with a two-sided $(95 \% \mathrm{CI})$ Wald test using the Benjamini-Hochberg method for multiple comparisons. b In PBMCs at d13 post ATI, the distribution of the differentiation subsets was measured by flow cytometry as a frequency of NK cells: Stage 0 (red, NKG2a/ $\mathrm{C}^{\text {low } C D 16}{ }^{-}$), Stage 1 (blue, $\mathrm{NKG2a} / \mathrm{c}^{\text {high }} \mathrm{CD} 16^{-}$), Stage 2 (orange, NKG2a/ $\mathrm{c}^{\text {high }} \mathrm{CD} 16^{+}$), and Stage 3 (purple, NKG2a/ ${ }^{\text {low }} \mathrm{CD} 16^{+}$). NK cells isolated from PBMCs at d13 post ATI were utilized to determine $\mathbf{c}$ the frequency of ex vivo innate activity (CD107a surface expression) or $\mathbf{d}$ the Env-specific activity upon co-culture with K562 cells expressing HLA-E loaded with SIVmac Env peptides. b-d Data from individual cytokine-treated (PEG-IFN $\alpha$ with prior ART + IL-21 + rIFN $\alpha$, blue; $n=8)$ and control (prior ART-only, black; $n=5$ ) RMs (open symbols) are overlaid against the mean \pm SEM (in red) and were analyzed with $\mathbf{b}, \mathbf{c}$ two-sided (95\% $\mathrm{Cl}$ ), two-way ANOVA with Bonferroni's correction for cross-sectional comparisons relative to controls or $\mathbf{d}$ with a two-sided (95\% $\mathrm{Cl}$ ) Mann-Whitney $\mathrm{U}$ test. e The delay in the rebound of plasma viremia was correlated against measures of SIV content, NK cell differentiation, and NK cell activity (as indicated at left; $n=13$ ) from the final on-ART measurement (d339-374 p.i.) or during rebound following ATI (d6-13 post ATI). Per each correlation the two-tailed $(95 \% \mathrm{Cl})$ Spearman's rank correlation coefficient (rho) is represented as a double-gradient heatmap and the size of each data point corresponds inversely to the $\log _{10}$-transformed Spearman's $p$ value. The false discovery rates (FDR) were calculated using SAS and significant values (Q $\left.<0.05\right)$ are represented by a black border. Correlations for which data did not exist during that experimental phase are indicated as "NA".

were segmented using a sterile scalpel; macerated over RPMI 1640 medium supplemented with $10 \%$ heat-inactivated fetal bovine serum (FBS; Gemini Bio), $100 \mathrm{U} /$ $\mathrm{mL}$ penicillin, and $100 \mu \mathrm{g} / \mathrm{mL}$ streptomycin; and filtered through a $100-\mu \mathrm{m}$ strainer to isolate mononuclear cells. Tissue segments of $\mathrm{LN}$ and 2-4 RB punches were flash frozen in dry ice for SIV-DNA analysis, whereas processed mononuclear cells were cryo-preserved in 10\% dimethyl sulfoxide (DMSO) in FBS.

Flow cytometric analysis. Fourteen-parameter flow cytometric analysis was performed on fresh PBMCs and mononuclear cells derived from LN biopsies and RB punches. Samples were stained utilizing standard procedures employing clones of anti-human mAbs that we have shown to be cross-reactive in $\mathrm{RMs}^{20,44,46,47}$ and are validated in databases maintained by NHP Reagent Resource (MassBiologics: https:// www.nhpreagents.org/ReactivityDatabase). The following antibodies were utilized for the longitudinal staining panel per T-cell and B-cell characterization: anti-CD21-PE (clone B-ly4, $10 \mu \mathrm{L}$, cat. 555422), anti-CD28-PE-CF594 (clone CD28.2, $5 \mu \mathrm{L}$, cat. 562296), anti-CD95-PE-Cy5 (clone DX2, $10 \mu \mathrm{L}$, cat. 559773), anti-CCR7-PE-Cy7 (clone 3D12, 7.5 $\mu \mathrm{L}$, cat. 557648), anti-CD45RA-APC (clone 5H9, $10 \mu \mathrm{L}$, cat. 561210), anti-Ki-67-AL700 (clone B56, $5 \mu \mathrm{L}$, cat. 561277), and anti-CD3-APC-Cy7 (clone SP34-2, $5 \mu \mathrm{L}$, cat. 557757) all from BD Biosciences; anti-HLA-DR-BV570 (clone L243, $5 \mu \mathrm{L}$, cat. 307638), anti-CD27-BV605 (clone O323, $10 \mu \mathrm{L}$, cat. 302830), anti-CD20PerCP-Cy5.5 (clone 2H7, $5 \mu \mathrm{L}$, cat. 302326), and anti-CD4-BV421 (clone OKT4, 2.5 $\mu \mathrm{L}$, cat. 317438) all from BioLegend; anti-CD38-FITC (clone AT-1, $5 \mu \mathrm{L}$, cat. 60131FI) from STEMCELL Technologies; and anti-CD8-Qdot705 (clone 3B5, $1 \mu \mathrm{L}$, cat. Q10059) and Live/Dead Fixable Aqua (AmCyan, $2 \mu \mathrm{L}$ of 1:20 PBS dilution, cat. L34957) from Thermo Fisher Scientific (Supplementary Fig. 1a). A secondary panel using a similar parental gating strategy, with the exception of using anti-CD4-BV605 (clone OKT4, $5 \mu \mathrm{L}$, cat. 317438 from BioLegend), was utilized to measure markers of cytotoxicity, such as anti-T-bet-PE (clone eBio4B10, $5 \mu \mathrm{L}$, cat. 12-5825-82 from eBioscience; Supplementary Fig. 1d). Stain volumes are given per $10^{6}$ live mononuclear cells or per 4-5 processed RB punches. Cells were fixed and permeabilized with a Cytofix/Cytoperm kit (BD Biosciences). Flow cytometry acquisition was performed on a minimum of 120,000 live $\mathrm{CD}^{+} \mathrm{T}$ cells for PBMC and $\mathrm{LN}$, and a minimum of 50,000 live $\mathrm{CD}^{+} \mathrm{T}$ cells in RB. Data acquisition was performed on an LSR II (BD Biosciences) using FACS DiVa software (v8.0.1) and analyzed using FlowJo software (version 9.9.6; TreeStar). Gates for activation (HLA-DR ${ }^{+} \mathrm{CD} 38^{+}$) and proliferation in memory $\mathrm{CD} 4^{+} \mathrm{T}$ cells and memory $\mathrm{CD} 8^{+} \mathrm{T}$ cells were set based on their expression within the corresponding naive subset.

Cryo-preserved PBMCs were stained as previously described ${ }^{37}$ to characterize NK cell subsets using a panel of the following mAbs: anti-CD3-V500 (clone SP34-2, $5 \mu \mathrm{L}$, cat. 560770), anti-CD45-PerCP (clone D058-1283, $5 \mu \mathrm{L}$, cat. 558411), and anti-CD69APC-Cy7 (clone FN50, $5 \mu \mathrm{L}$, cat 557756) all from BD Biosciences; anti-CD8-VioBlue (clone BW135/80, $2.5 \mu \mathrm{L}$, cat. 130-094-152), anti-NKp80-FITC (clone 4A4.D10, $8 \mu \mathrm{L}$, cat. 130-094-843), anti-NKp30-APC (clone AF29-4D12, $8 \mu \mathrm{L}$, cat. 130-092-484) all from Miltenyi Biotec; and anti-CD20-AL700 (clone 2H7, $8 \mu \mathrm{L}$, cat. 56-0209) from eBioscience; anti-TIM-3-PE-Cy7 (clone F38-2E2, $5 \mu \mathrm{L}$, cat. 345014); and anti-NKG2aPE (clone Z199, $8 \mu \mathrm{L}$, cat. IM3291U), anti-CD16-ECD (clone 3G8, $5 \mu \mathrm{L}$, cat. 6607111), and anti-NKp46-PE-Cy5 (clone BAB281, $8 \mu \mathrm{L}$, cat. A66902) all from Beckman Coulter (Supplementary Fig. 8). A secondary immunophenotyping panel consisted of the following mAbs: anti-CD3-V500 (clone SP34-2, $5 \mu \mathrm{L}$, cat. 560770), anti-CD45-PerCP (clone D058-1283, $5 \mu \mathrm{L}$, cat. 558411), anti-CD95-APC (clone DX2, $5 \mu \mathrm{L}$, cat. 558814), anti-CD107a-PE-Cy5 (clone HA43, $5 \mu \mathrm{L}$, cat. 555802), and anti-CCR7-BV711 (clone 3D12, $5 \mu \mathrm{L}$, cat. 563712) all from BD Biosciences; anti-CD8-VioBlue (clone BW135/80, $2.5 \mu \mathrm{L}$, cat. 130-094-152) from Miltenyi Biotec; anti-CD16-ECD (clone 3G8, $5 \mu \mathrm{L}$, cat. 41116015) and anti-NKG2a-PE (clone Z199, $8 \mu \mathrm{L}$, cat. IM3291U) from Beckman Coulter; anti-HLA-DR-PE-Cy7 (clone L243, $5 \mu \mathrm{L}$, cat. 307616) and anti-PD-1-APC Cy7 (clone EH12.2H7, $5 \mu \mathrm{L}$, cat. 329922) from BioLegend; and anti-CXCR5-FITC (clone MU5UBEE, $5 \mu \mathrm{L}$, cat. 15566616) from eBioscience. A variant of this panel used anti-HLA-E-PE (clone 3D12HLA-E, $6 \mu \mathrm{L}$, cat. NBP2-00277) from R\&D Systems. Representative gating strategies are given in Supplementary Fig. 1b, $\mathrm{c}$ and representative CD107a stains are given in Supplementary Fig. 6d-f. The anti-NKG2a mAb recognizes both NKG2a and NKG2c in macaques. Stain volumes are given per $10^{6}$ live 
mononuclear cells upon thawing. Cells were fixed and permeabilized with a Cytofix/ Cytoperm kit (BD Biosciences) and intracellular stain was incubated a $4^{\circ} \mathrm{C}$ for $15 \mathrm{~min}$ and anti-IFN- $\gamma$-AL700 (clone GB11, $8 \mu \mathrm{L}$, cat. 560213). For the NK cell panels, flow cytometry acquisitions were done on a LSR II (BD Biosciences) and were analyzed using FlowJo software (version 10.4.2; TreeStar).

Intracellular cytokine staining. Th17 and Th22 cells were defined as the frequency IL-17 and IL-22 producing CD4 $4^{+}$T cells upon ex vivo stimulation with PMA and ionomycin ${ }^{20,22}$. Fresh mononuclear cells derived from RB punches were incubated for $4 \mathrm{~h}$ at $37^{\circ} \mathrm{C}$ in RPMI 1640 medium supplemented with $10 \%$ FBS containing PMA $(80 \mathrm{ng} / \mathrm{mL}$; cat. P8139, Millipore Sigma), calcium ionophore A23187 (500 ng/mL; cat. C9275, Millipore Sigma), brefeldin A (10 $\mu \mathrm{g} / \mathrm{mL}$; cat. 420601, BioLegend), and GolgiStop with monensin $\left(7 \times 10^{-4}\right.$ dilution; cat. 554724, BD Biosciences). Samples were stained utilizing standard procedures employing clones of anti-human mAbs that were cross-reactive in RMs as validated by NHP Reagent Resource (MassBiologics): anti-CD8-PE-CF594 (clone RPA-T8, clone 562282), anti-CD95-PE-Cy5 (clone DX2, $10 \mu \mathrm{L}$, cat. 559773), anti-IFN $\gamma$-PE-Cy7 (clone B27, $5 \mu \mathrm{L}$, cat. 557643), anti-TNFaAL700 (clone Mab11, $1 \mu \mathrm{L}$, cat. 557996), and anti-CD3-Cy7APC (clone SP34-2, $5 \mu \mathrm{L}$, cat. 557757) all from BD Biosciences; anti-CD4-BV421 (clone OKT4, $4 \mu \mathrm{L}$, cat. 317434) and anti-IL-2-BV605 (clone MQ1-17H12, $1 \mu \mathrm{L}$, cat. 500332) both from BioLegend; and anti-IL-17-FITC (clone eBio64DEC17, $5 \mu \mathrm{L}$, cat. 53-7179-42), anti-IL22-APC (clone IL22JOP, $5 \mu \mathrm{L}$, cat. 17-7222-82), and Live/Dead Fixable Aqua (AmCyan, $2 \mu \mathrm{L}$ of 1:20 PBS dilution, cat. L34957) from Thermo Fisher Scientific (Supplementary Fig. 3a). Stain volumes are given per test (approximately 5-10 RB punches). Cells were fixed and permeabilized with a Cytofix/Cytoperm kit (BD Biosciences). The intracellular stain was incubated for $1 \mathrm{~h}$ at room temperature in the dark. Data acquisition was performed on a minimum of 50,000 live $\mathrm{CD}^{3}+\mathrm{T}$ cells on an LSR II (BD Biosciences) using FACS DiVa software (v8.0.1) and analyzed using FlowJo software (version 9.9.6; TreeStar).

Determination of plasma viremia. Six-replicate reaction hybrid qRT-PCR assay was performed to determine SIV-RNA copies per $\mathrm{mL}$ of EDTA plasma (i.e., viral loads) as previously described ${ }^{48}$ with a limit of detection of 30 copies $/ \mathrm{mL}$. While primers target a highly conserved viral sequence in $\mathrm{SIVmac}_{239}$ gag (Supplementary Table 2), unconventional neutral bases are incorporated to avoid biasing in measuring off-target viral sequences. A Poisson method was employed to calculate values for which not all reactions were positive, which was otherwise calculated by interpolating individual cycle threshold values on a standard curve. Primers and probe sequences are given in Supplementary Table 2.

Quantification of cell-associated SIV-DNA and -RNA. Analyses were performed on cryo-preserved PBMCs, snap frozen LN biopsies, and snap frozen RB punches. Cell-associated SIV-DNA and -RNA were extracted with an AllPrep DNA/RNA Mini Kit (Qiagen), and were quantified via hybrid real-time/digital qPCR and RTqPCR assays for SIV-DNA and -RNA, respectively, in ten replicates with singlecopy clinical sensitivity as previously described ${ }^{49}$. Viral copy numbers were normalized based on genomic CCR5. A Poisson method was employed to calculate samples for which there were fewer than ten positive replicates for amplification, which was otherwise calculated by interpolating a standard curve. Primers and probe sequences are given in Supplementary Table 2.

Quantitative viral outgrowth assay (QVOA). Restricted by cellular yields, the QVOA was limited to four controls (ART-only) and six cytokine-treated RMs (ART + IL-21 + IFNa). CD4 ${ }^{+}$cells were positively selected utilizing a MACS CD4 MicroBeads kit for NHPs (cat. 130-091-102, Miltenyi Biotec) from LN cryo-preserved mononuclear cells. After a $1 \mathrm{~h}$ rest in RPMI 1640 medium supplemented with $10 \%$ FBS, cells were stimulated for $16 \mathrm{~h}$ at $37^{\circ} \mathrm{C}$ with IL-2 (recombinant human, $20 \mathrm{ng} / \mathrm{mL}$; Tonbo Biosciences), anti-CD28 mAb (clone CD28., $5 \mu \mathrm{g} / \mathrm{mL}$; BD Pharmingen), and anti-CD2 mAb (clone RPA-2.10, $5 \mu \mathrm{g} / \mathrm{mL}$; BioLegend) in a 96-well polystyrene plate coated with anti-CD $3 \mathrm{mAb}$ (clone SP34-2, $1 \mu \mathrm{g}$ per well; BD Biosciences). Stimulated $\mathrm{CD}^{+}$cells were washed twice and co-cultured in a 1:1 ratio with 174xCEM cell line (HIV Reagent Program managed by ATCC) ${ }^{50}$ in three serial dilutions in triplicates ranging from $1 \times 10^{6}$ to $0.1 \times 10^{6}$ cells per well. The cells were cultured at $37^{\circ} \mathrm{C}$ under $5 \% \mathrm{CO}_{2}$ in complete RPMI 1640 with $4 \mathrm{mM} \mathrm{L-glutamine} \mathrm{supplemented} \mathrm{with} 10 \%$ heat-inactivated FBS (Gemini Bio-products), penicillin $(50 \mathrm{U} / \mathrm{mL})$, streptomycin $(50$ $\mu \mathrm{g} / \mathrm{mL})$, and IL-2 $(20 \mathrm{ng} / \mathrm{mL})$. Cultured cells were fed weekly with fresh medium supplemented with IL-2 $(20 \mathrm{ng} / \mathrm{mL}$ final concentration), and harvested and analyzed at day 10 and 25 . Positive wells were identified by the expression of intracellular SIVGag p27 via flow cytometry ${ }^{51}$ and the amplification $(>10 \times)$ of SIV-RNA (copies/mL) in the supernatant as determined by 6-replicate reaction qRT-PCR between day 10 and 25 . The frequency of cells producing replication competent virus was determined by the maximum-likelihood method ${ }^{52}$ utilizing IUPMStats ${ }^{53}$ and was expressed in infectious units per million.

Quantification of IFN- $\gamma$ response upon SIV-Gag and -Env stimulation. ELISpots were performed as previously described ${ }^{46}$. Briefly, cryo-preserved PBMCs were thawed, rested, and triplicate plated $\left(4 \times 10^{5}\right.$ cells per well $)$ for an enzyme-linked immune absorbent spot (ELISpot) assay. Cells were either mock stimulated (DMSO); stimulated with overlapping SIVmac 239 Gag or Env peptides (HIV
Reagent Program managed by ATCC; $1 \mu \mathrm{g} / \mathrm{mL}$ ); or stimulated with Concavalin A (Sigma; $2.5 \mu \mathrm{g} / \mathrm{mL}$ ). Plates were incubated for $24 \mathrm{~h}$ at $37^{\circ} \mathrm{C}$ and the number of IFN$\gamma$ secreting cells was quantified per the manufacturer's instructions using the Monkey IFN- $\gamma$ ELISpot ${ }^{\text {PLUS }}$ kit (MABTECH). Spot forming units (SFU) per well were counted on an ELISpot plate reader and represented as SFU per million cells The IFN- $\gamma$ response given per stimulation condition is an average of triplicate reactions with the average DMSO background subtracted.

Ex vivo innate NK cell activity assay. NK cell degranulation activity was determined through expression of cell surface CD107a, as previously described ${ }^{54}$. Cryopreserved PBMCs were labeled with anti-NKG2a/c-PE (clone Z199, cat. IM3291U; Beckman Coulter) conjugated with anti-PE MicroBeads (Miltenyi Biotec) and magnetically isolated according to the manufacturer's instructions. NKG2a/c ${ }^{+}$cells were incubated overnight at $37^{\circ} \mathrm{C}$ with $5 \% \mathrm{CO}_{2}$ in RPMI supplemented with $10 \%$ (v/v) FBS, $100 \mathrm{U} / \mathrm{mL} \mathrm{IL-2,} \mathrm{and} 10 \mathrm{ng} / \mathrm{mL}$ of IL-15, and then analyzed for cell surface $\mathrm{CD} 107 \mathrm{a}$ expression via flow cytometry.

Env-specific NK cell activity assay. K562 cells devoid of MHC-I stably (ATCC) expressing HLA-E*0101 (K562-E*0101; Applied Biological Materials Inc.) cells were incubated with $50 \mu \mathrm{M}$ of $\operatorname{SIVmac}_{239 / 251}$ Env peptide (NQLLIAILL) at $26^{\circ} \mathrm{C}$ for $15-20 \mathrm{~h}^{7}$. Isolated NK cells were cultured for $6 \mathrm{~h}$ in the presence of an antiCD107a-PE-Cy5 mAb (clone HA43, $5 \mu \mathrm{L}$, cat. 555802; BD Biosciences), either alone (NK), or co-cultured at a 5:1 ratio with $2 \times 10^{4} \mathrm{~K} 562-\mathrm{E}^{*} 0101$ cells that were either unpulsed $\left(\mathrm{NK}+\mathrm{K} 562^{*} \mathrm{E}\right)$ or loaded with SIVmac $239 / 251$ Env peptide $\left(\mathrm{NK}+\mathrm{K} 563^{*} \mathrm{E}+\mathrm{ENV}\right)$. GolgiStop and GolgiPlug (BD Biosciences) was added $1 \mathrm{~h}$ following culture initiation. The frequency of NK cells expressing surface CD107a was measured by flow cytometry per each culture condition to assess the

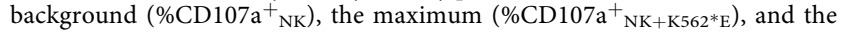
peptide-specific degranulation activity $\left(\% \mathrm{CD} 107 \mathrm{a}^{+} \mathrm{NK}+\mathrm{K} 562^{*} \mathrm{E}+\mathrm{ENV}\right.$; Supplementary Fig. 6). From these measurements the Env-specific NK cell activity was calculated as previously described ${ }^{30,55}$ :

$$
\log _{10}\left[100 \times \frac{\% \mathrm{CD} 107 \mathrm{a}_{\mathrm{NK}+\mathrm{K} 562^{*} \mathrm{E}+\mathrm{ENV}}^{+}-\% \mathrm{CD} 107 \mathrm{a}_{\mathrm{NK}}^{+}}{\% \mathrm{CD} 107 \mathrm{a}_{\mathrm{NK}+\mathrm{K} 562^{*} \mathrm{E}}^{+}-\% \mathrm{CD} 107 \mathrm{a}_{\mathrm{NK}}^{+}}\right]=\text {Env }- \text { specific NK cell activity }
$$

Production and testing of rhesus rIL-21-IgFc. A fusion protein of rhesus IL-21$\mathrm{IgFc}$ (IL-21) fusion protein was generated as previously described by the Resource of Nonhuman Primate Immune Reagents at New Iberia Research Center and were provided via MTA ${ }^{20,22,56,57}$. Using the Drosophila S2 system, a fusion protein was produced between rMamuIL-21 and a macaque $\operatorname{IgG} 2 \mathrm{Fc}$, which was mutated (L235A and P331S) to block complement or Fc receptor binding ${ }^{58,59}$. IL-21 was isolated to $>95 \%$ purity by Protein-G sepharose affinity chromatography, dialyzed against PBS, and tested for sterility and confirmed endotoxin free.

Production and testing of rhesus IFN $\boldsymbol{\alpha}$-IgFc. Using the Drosophila S2 system, a fusion protein was produced, as previously described ${ }^{56,57}$, between a recombinant RM IFNa2 and a macaque IgG2 $\mathrm{Fc}^{19}$ by the Resource of Nonhuman Primate Immune Reagents at New Iberia Research Center and was provided via MTA. The Fc was mutated at two positions (L235A and P331S) to block complement or Fc receptor binding 58,59 . IFNa-IgFc was isolated to $>95 \%$ purity by Protein-G sepharose affinity chromatography, dialyzed against PBS, lyophilized, and tested for sterility and confirmed endotoxin free. Activity was verified as $264 \times 10^{3} \mathrm{U} / \mathrm{mg}$ as determined by Vero/EMCV bioassay.

Peginterferon alfa-2A. Pharmaceutical-grade human PEG-IFNa was purchased at cost (PEGASYS ${ }^{\circledast}$, Roche), which was previously shown to be well tolerated in SIVinfected macaques ${ }^{21,60}$.

RNA sequencing. RNA was extracted from PBMCs stored at $-80^{\circ} \mathrm{C}$ in RLT buffer with 1\% 2-mercaptoethanol using RNeasy Mini kits (QIAGEN, CA) with DNase digest and QIAcube automation stations. Extracted RNA was quantified using a NanoDrop 2000 spectrophotometer (Thermo Scientific Inc. Wilmington, DE) and the quality was assessed by Bioanalyzer analysis (Agilent Technologies, Santa Clara, CA). Ten nanogram of total RNA was used as input for cDNA amplification using $5^{\prime}$ template-switch PCR with the Clontech SMART-Seq v4 Ultra Low Input RNA kit. Amplified cDNA was fragmented and appended with dual indexed bar codes using Illumina NexteraXT DNA Library Prep kits. The amplified libraries from both sets were validated by capillary electrophoresis on the Agilent 4200 TapeStation. The libraries were normalized, pooled, and sequenced on the Illumina HiSeq 3000 system employing a single-end 101 cycles run at average read depths of $30 \times 10^{6}$ reads per sample. Reads were mapped to the MacaM version 7 assembly of the Indian rhesus macaque genomic reference ${ }^{61}$ with RhesusGenome using STAR (version 2.5.2b) with default alignment parameters ${ }^{62}$ (https://www.unmc.edu/ rhesusgenechip/index.htm). Abundance estimation of raw read counts per transcript was done internally with STAR using the HTSeq-count algorithm ${ }^{63}$.

Statistics and reproducibility. Statistical tests were two-sided and $p$ values $\leq 0.05$ ( $95 \%$ confidence interval, CI) were considered statistically significant for each of the 
specific statistical comparisons. All experiments were performed as a single technical replicate unless otherwise noted in the Methods (i.e., qRT-PCR, IFN- $\gamma$ ELISpot, and QVOA). No assays were repeated as independent experiments. Data were tested for Gaussian distribution using the D'Agostino-Pearson omnibus normality test. Data showing continuous outcomes are represented as mean \pm SEM. Two-way ANOVAs and/or mixed-effects models, in the event of absent data points, were performed with Bonferroni's correction for multiple comparisons. Correlations were performed twosided with a non-parametric Spearman correlation and were fitted with a simple linear regression. Comparisons of survival curves were conducted with a Log-rank (Mantel-Cox) test. All of the above analyses were conducted using GraphPad Prism version 8.1.2. Using SAS, Spearman's $p$ values were adjusted for multiple comparisons using the stepdown Bonferroni ${ }^{64}$, Hochberg ${ }^{65}$, and false discovery rate (FDR) ${ }^{66}$ methods. Correlation and RNA-seq data were visualized using ggplot2 (version 3.3.2) in RStudio (version 1.4.1103) with custom code. The distribution of cytokine coexpression (i.e., Boolean logical gates of IL-17 and IL-22 expression within CD4 ${ }^{+}$Tcells) was analyzed with a Permutation test $\left(10^{3}\right.$ iterations) in SPICE version $6^{67}$. Rates of increase in $\log _{10}$ SIV-RNA copies per $\mathrm{mL}$ of plasma post ATI were obtained using a mixed-effects linear model specifying that data follow a linear regression over time, with a random intercept for each animal. The mean slope and mean linear increase were estimated and compared between treatment conditions within the framework of the mixed-effects linear model ${ }^{68}$. DESeq 2 version $1.22 .1 \mathrm{R}$ package ${ }^{69}$ was used to produce normalized read counts and compute the differential expression estimation using the Wald test. Multiple-test correction was performed with the Benjamini-Hochberg method and a FDR $<0.05$ was used to indicate statistical significance.

Reporting summary. Further information on research design is available in the Nature Research Reporting Summary linked to this article.

\section{Data availability}

Materials provided via MTA were supplied without restrictions on use. Source data are provided for the performed correlations, as are the statistical readouts for the correlations and RNA-seq analyses. RNA-seq data related to Figs. If and 5a, and Supplementary Fig. 4 are publicly available in GenBank (https://www.ncbi.nlm.nih.gov/geo/query/acc. cgi) under GEO accession GSE163443 with subseries GSE163440, GSE163441, and GSE163442. The MacaM version 7 assembly of the Indian rhesus macaque genomic reference is publicly available at https://www.unmc.edu/rhesusgenechip/index. htm. Source Data are provided with this paper.

\section{Code availability}

Other data that support the findings of this work, including custom ggplot2 (version 3.3.2) code for data visualization (RStudio version 1.4.1103) of Figs. 1f, $2 \mathrm{~h}, 3 \mathrm{j}, 5 \mathrm{a}, \mathrm{e}$, and Supplementary Fig. 4, are available from the corresponding author on reasonable request. Source Data are provided with this paper.

Received: 28 August 2020; Accepted: 8 April 2021; Published online: 17 May 2021

\section{References}

1. Kim, S. et al. Licensing of natural killer cells by host major histocompatibility complex class I molecules. Nature 436, 709-713 (2005).

2. Houchins, J. P. et al. Natural killer cell cytolytic activity is inhibited by NKG2A and activated by NKG2-C. J. Immunol. 158, 3603-3609 (1997).

3. Carretero, M. et al. The CD94 and NKG2-A C-type lectins covalently assemble to form a natural killer cell inhibitory receptor for HLA class I molecules. Eur. J. Immunol. 27, 563-567 (1997).

4. Braud, V. M. et al. HLA-E binds to natural killer cell receptors CD94/NKG2A, B and C. Nature 391, 795-799 (1998).

5. Ramsuran, V. et al. Elevated HLA-A expression impairs HIV control through inhibition of NKG2A-expressing cells. Science 359, 86-90 (2018).

6. Nattermann, J. et al. HIV-1 infection leads to increased HLA-E expression resulting in impaired function of natural killer cells. Antivir. Ther. 10, 95-107 (2005).

7. Huot, N. et al. SIV-induced terminally differentiated adaptive NK cells in lymph nodes associated with enhanced MHC-E restricted activity. Nat. Commun. 12, 1282 (2021).

8. Nimmerjahn, F. \& Ravetch, J. V. Fcgamma receptors as regulators of immune responses. Nat. Rev. Immunol. 8, 34-47 (2008).

9. Parrish-Novak, J. et al. Interleukin 21 and its receptor are involved in NK cell expansion and regulation of lymphocyte function. Nature 408, 57-63 (2000).

10. Kasaian, M. T. et al. IL-21 limits NK cell responses and promotes antigenspecific $\mathrm{T}$ cell activation: a mediator of the transition from innate to adaptive immunity. Immunity 16, 559-569 (2002).

11. Seo, H. et al. IL-21-mediated reversal of NK cell exhaustion facilitates anti-tumour immunity in MHC class I-deficient tumours. Nat. Commun. 8, 15776 (2017)
12. Hansen, M. L. et al. IFN-alpha primes T- and NK-cells for IL-15-mediated signaling and cytotoxicity. Mol. Immunol. 48, 2087-2093 (2011).

13. Tomescu, C., Tebas, P. \& Montaner, L. J. IFN-alpha augments natural killermediated antibody-dependent cellular cytotoxicity of HIV-1-infected autologous CD4+ T cells regardless of major histocompatibility complex class 1 downregulation. AIDS 31, 613-622 (2017).

14. Kwaa, A. K. R., Talana, C. A. G. \& Blankson, J. N. Interferon alpha enhances NK cell function and the suppressive capacity of HIV-specific CD8+ T cells. J. Virol. 93, e01541-18 (2019).

15. Strengell, M., Julkunen, I. \& Matikainen, S. IFN-alpha regulates IL-21 and IL21R expression in human NK and T cells. J. Leukoc. Biol. 76, 416-422 (2004).

16. Papasavvas, E. et al. NK response correlates with HIV decrease in pegylated IFN-alpha2a-treated antiretroviral therapy-suppressed subjects. J. Immunol. 203, 705-717 (2019).

17. Jacquelin, B. et al. Innate immune responses and rapid control of inflammation in African green monkeys treated or not with interferon-alpha during primary SIVagm infection. PLoS Pathog. 10, e1004241 (2014).

18. Del Prete, G. Q. et al. Short communication: comparative evaluation of coformulated injectable combination antiretroviral therapy regimens in simian immunodeficiency virus-infected rhesus macaques. AIDS Res. Hum. Retroviruses 32, 163-168 (2016).

19. Vanderford, T. H. et al. Treatment of SIV-infected sooty mangabeys with a type-I IFN agonist results in decreased virus replication without inducing hyperimmune activation. Blood 119, 5750-5757 (2012).

20. Micci, L. et al. Interleukin-21 combined with ART reduces inflammation and viral reservoir in SIV-infected macaques. J. Clin. Invest 125, 4497-4513 (2015).

21. Palesch, D. et al. Short-term pegylated interferon alpha2a treatment does not significantly reduce the viral reservoir of simian immunodeficiency virusinfected, antiretroviral therapy-treated rhesus macaques. J. Virol. 92, e00279-18 (2018)

22. Pallikkuth, S. et al. Maintenance of intestinal Th17 cells and reduced microbial translocation in SIV-infected rhesus macaques treated with interleukin (IL) -21. PLoS Pathog. 9, e1003471 (2013).

23. Dunham, R. M. et al. Pharmacologic inhibition of IDO blunts features of SIVrelated chronic inflammation. In Conference on Retroviruses and Opportunistic Infections (Seattle, WA, 2017).

24. Jacquelin, B. et al. Nonpathogenic SIV infection of African green monkeys induces a strong but rapidly controlled type I IFN response. J. Clin. Invest 119 3544-3555 (2009).

25. Bosinger, S. E. et al. Global genomic analysis reveals rapid control of a robust innate response in SIV-infected sooty mangabeys. J. Clin. Invest 119, 3556-3572 (2009)

26. Szabo, S. J. et al. A novel transcription factor, T-bet, directs Th1 lineage commitment. Cell 100, 655-669 (2000)

27. d'Ettorre, G. et al. Reconstitution of intestinal CD4 and Th17 T cells in antiretroviral therapy suppressed HIV-infected subjects: implication for residual immune activation from the results of a clinical trial. PLoS One 9 e109791 (2014)

28. Li, J. Z. et al. ART reduces T cell activation and immune exhaustion markers in HIV controllers. Clin. Infect. Dis. 70, 1636-1642 (2019).

29. Reuter, M. A. et al. HIV-specific CD8 $(+) \mathrm{T}$ cells exhibit reduced and differentially regulated cytolytic activity in lymphoid tissue. Cell Rep. 21, 3458-3470 (2017)

30. Alter, G., Malenfant, J. M. \& Altfeld, M. CD107a as a functional marker for the identification of natural killer cell activity. J. Immunol. Methods 294, 15-22 (2004).

31. Azzoni, L. et al. Pegylated Interferon alfa-2a monotherapy results in suppression of HIV type 1 replication and decreased cell-associated HIV DNA integration. J. Infect. Dis. 207, 213-222 (2013).

32. Boue, F. et al. Alpha interferon administration during structured interruptions of combination antiretroviral therapy in patients with chronic HIV-1 infection: INTERVAC ANRS 105 trial. AIDS 25, 115-118 (2011).

33. Paust, S. et al. Critical role for the chemokine receptor CXCR6 in NK cellmediated antigen-specific memory of haptens and viruses. Nat. Immunol. 11, 1127-1135 (2010)

34. O’Leary, J. G., Goodarzi, M., Drayton, D. L. \& von Andrian, U. H. T cell- and B cell-independent adaptive immunity mediated by natural killer cells. Nat. Immunol. 7, 507-516 (2006).

35. Smith, H. R. et al. Recognition of a virus-encoded ligand by a natural killer cell activation receptor. Proc. Natl Acad. Sci. USA 99, 8826-8831 (2002)

36. Wang, Y. et al. HIV-1-induced cytokines deplete homeostatic innate lymphoid cells and expand TCF7-dependent memory NK cells. Nat. Immunol. 21, 274-286 (2020).

37. Huot, N. et al. Natural killer cells migrate into and control simian immunodeficiency virus replication in lymph node follicles in African green monkeys. Nat. Med. 23, 1277-1286 (2017).

38. Huot, N. et al. Lymph node cellular and viral dynamics in natural hosts and impact for HIV cure strategies. Front Immunol. 9, 780 (2018). 
39. Raehtz, K. D. et al. African green monkeys avoid SIV disease progression by preventing intestinal dysfunction and maintaining mucosal barrier integrity. PLoS Pathog. 16, e1008333 (2020).

40. Cartwright, E. K. et al. CD8 $(+)$ lymphocytes are required for maintaining viral suppression in SIV-infected macaques treated with short-term antiretroviral therapy. Immunity 45, 656-668 (2016).

41. McBrien, J. B. et al. Robust and persistent reactivation of SIV and HIV by N803 and depletion of CD8(+) cells. Nature 578, 154-159 (2020).

42. Cao, Y., Cartwright, E. K., Silvestri, G. \& Perelson, A. S. CD8+ lymphocyte control of SIV infection during antiretroviral therapy. PLoS Pathog. 14, e1007350 (2018).

43. Webster, R. L. \& Johnson, R. P. Delineation of multiple subpopulations of natural killer cells in rhesus macaques. Immunology 115, 206-214 (2005).

44. McGary, C. S. et al. CTLA-4+PD-1- memory CD4+ T cells critically contribute to viral persistence in antiretroviral therapy-suppressed, SIVinfected rhesus macaques. Immunity 47, 776-788.e775 (2017).

45. Strongin, Z. et al. Virologic and immunologic features of simian immunodeficiency virus control post-ART interruption in rhesus macaques. J. Virol. 94, e00338-20 (2020).

46. Harper, J. et al. CTLA-4 and PD-1 dual blockade induces SIV reactivation without control of rebound after antiretroviral therapy interruption. Nat. Med. 26, 519-528 (2020).

47. Huot, N. et al. Non-human primate determinants of natural killer cells in tissues at steady-state and during simian immunodeficiency virus infection. Front Immunol. 11, 2134 (2020).

48. $\mathrm{Li}, \mathrm{H}$. et al. Envelope residue 375 substitutions in simian-human immunodeficiency viruses enhance CD4 binding and replication in rhesus macaques. Proc. Natl Acad. Sci. USA 113, E3413-E3422 (2016).

49. Hansen, S. G. et al. Addendum: immune clearance of highly pathogenic SIV infection. Nature 547, 123-124 (2017).

50. Salter, R. D., Howell, D. N. \& Cresswell, P. Genes regulating HLA class I antigen expression in T-B lymphoblast hybrids. Immunogenetics 21, 235-246 (1985).

51. Fukazawa, Y. et al. B cell follicle sanctuary permits persistent productive simian immunodeficiency virus infection in elite controllers. Nat. Med. 21, 132-139 (2015).

52. Myers, L. E., McQuay, L. J. \& Hollinger, F. B. Dilution assay statistics. J. Clin. Microbiol. 32, 732-739 (1994).

53. Rosenbloom, D. I. et al. Designing and interpreting limiting dilution assays: general principles and applications to the latent reservoir for human immunodeficiency virus-1. Open Forum Infect. Dis. 2, ofv123 (2015).

54. Bryceson, Y. T. et al. Functional analysis of human NK cells by flow cytometry. Methods Mol. Biol. 612, 335-352 (2010).

55. Reeves, R. K. et al. Antigen-specific NK cell memory in rhesus macaques. Nat. Immunol. 16, 927-932 (2015).

56. Onlamoon, N. et al. Soluble PD-1 rescues the proliferative response of simian immunodeficiency virus-specific CD4 and CD8 T cells during chronic infection. Immunology 124, 277-293 (2008).

57. Eberly, M. D. et al. Increased IL-15 production is associated with higher susceptibility of memory CD4 $\mathrm{T}$ cells to simian immunodeficiency virus during acute infection. J. Immunol. 182, 1439-1448 (2009).

58. Chappel, M. S. et al. Identification of the Fc gamma receptor class I binding site in human IgG through the use of recombinant IgG1/IgG2 hybrid and point-mutated antibodies. Proc. Natl Acad. Sci. USA 88, 9036-9040 (1991).

59. Xu, J., Green, P. C. \& Kemp, R. G. Identification of basic residues involved in substrate binding and catalysis by pyrophosphate-dependent phosphofructokinase from Propionibacterium freudenreichii. J. Biol. Chem. 269, 15553-15557 (1994).

60. Asmuth, D. M. et al. Pegylated interferon-alpha 2a treatment of chronic SIVinfected macaques. J. Med Primatol. 37, 26-30 (2008).

61. Zimin, A. V. et al. A new rhesus macaque assembly and annotation for nextgeneration sequencing analyses. Biol. Direct 9, 20 (2014).

62. Dobin, A. et al. STAR: ultrafast universal RNA-seq aligner. Bioinformatics 29, 15-21 (2013).

63. Anders, S., Pyl, P. T. \& Huber, W. HTSeq-a Python framework to work with high-throughput sequencing data. Bioinformatics 31, 166-169 (2015).

64. Holm, S. A simple sequentially rejective multiple test procedure. Scand. J. Stat. 6, 65-70 (1979).

65. Hochberg, Y. A sharper Bonferroni procedure for multiple tests of significance. Biometrika 75, 800-802 (1988).

66. Hochberg, Y. \& Benjamini, Y. Controlling the false discovery rate: a practical and powerful approach to multiple testing. J R Stat Soc Ser B 57, 289-300 (1995).

67. Roederer, M., Nozzi, J. L. \& Nason, M. C. SPICE: exploration and analysis of post-cytometric complex multivariate datasets. Cytom. A 79, 167-174 (2011).

68. Laird, N. M. \& Ware, J. H. Random-effects models for longitudinal data. Biometrics 38, 963-974 (1982).
69. Love, M. I., Huber, W. \& Anders, S. Moderated estimation of fold change and dispersion for RNA-seq data with DESeq2. Genome Biol. 15, 550 (2014).

\section{Acknowledgements}

We thank Stephanie Ehnert and Christopher Souder from Research Resources, and Sherrie Jean and Jennifer Wood from Veterinary Medicine at Yerkes National Primate Research Center (YNPRC) for providing animal and veterinary care, respectively. We gratefully acknowledge Katie Kitrinos at ViiV Healthcare for supplying DTG, and Romas Geleziunas at Gilead Sciences for supplying FTC and TDF. Both the rhesus rIL-21-IgFc and rhesus IFNa-IgFc were kindly supplied by Kenneth Rogers at the Resource for Nonhuman Primate Immune Reagents. We would also like to thank Mathias Lichterfeld, Pilar Garcia Broncano, and Xiaodong Lian of the Ragon Institute, and William Bosche at Leidos Biomedical Research for technical assistance. The SIVmac $\operatorname{man}_{239}$ strain used to infect the RMs was kindly provided by Koen Van Rompay of UC-Davis, and the 174xCEM cell line was supplied by Dr. Peter Cresswell via the HIV Reagent Program. We thank Richard Dunham at ViiV Healthcare for providing access to historical RNA-seq data from longitudinal SIV infection. This work was supported by the NIAID, NIH under award number R01AI116379 to M. P. and R01AI143457 to M. M.-T. Support for this work was also provided by ANRS and the Fondation J. Beytout to M. M.-T.; NCRR, NIH award 5R24RR016988 to F. V. and the Resource for Nonhuman Primate Immune Reagents; ORIP/OD award P51OD011132 to YNPRC; and NCI, NIH award HHSN261200800001E and 75N91019D00024 to Leidos Biomedical Research. P.R. was recipient of a PhD fellowship from the University Paris Diderot, Sorbonne Paris Cité. The content of this publication does not necessarily reflect the views or policies of the Department of Health and Human Services, nor does mention of trade names, commercial products, or organizations imply endorsement by the US Government.

\section{Author contributions}

J.H. contributed to conceptualization, methodology, formal analysis, investigation, writing (original draft, review, and editing), and visualization. N.H. performed the phenotyping and ex vivo functional assays on NK cells and contributed to conceptualization, methodology, investigation, data analyses, and writing (review and editing). L.M. contributed to conceptualization, methodology, and investigation. G.T., A.A. U., and S.B. performed RNA-seq analyses. C.K. and H.W. performed longitudinal processing and flow cytometry of tissues. P. R. contributed to methodology and investigation. C.G. performed the ELISpot experiments. N.S. and K.E. performed statistical analyses of the correlations and viral rebound kinetics. F.V. provided investigational compounds. J.L. measured virus content in plasma and tissue. G.S. contributed to conceptualization. B.J. contributed to methodology. M.M.-T. contributed to conceptualization, methodology, writing (review and editing), supervision, and funding acquisition. M.P. contributed to conceptualization, methodology, resources, writing (original draft, review, and editing), supervision, and funding acquisition.

\section{Competing interests}

The authors declare no competing interests.

\section{Additional information}

Supplementary information The online version contains supplementary material available at https://doi.org/10.1038/s41467-021-23189-7.

Correspondence and requests for materials should be addressed to M.P.

Peer review information Nature Communications thanks the anonymous reviewer(s) for their contribution to the peer review of this work. Peer reviewer reports are available.

Reprints and permission information is available at http://www.nature.com/reprints

Publisher's note Springer Nature remains neutral with regard to jurisdictional claims in published maps and institutional affiliations.

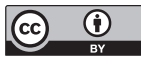

Open Access This article is licensed under a Creative Commons Attribution 4.0 International License, which permits use, sharing, adaptation, distribution and reproduction in any medium or format, as long as you give appropriate credit to the original author(s) and the source, provide a link to the Creative Commons license, and indicate if changes were made. The images or other third party material in this article are included in the article's Creative Commons license, unless indicated otherwise in a credit line to the material. If material is not included in the article's Creative Commons license and your intended use is not permitted by statutory regulation or exceeds the permitted use, you will need to obtain permission directly from the copyright holder. To view a copy of this license, visit http://creativecommons.org/ licenses/by/4.0/.

(c) The Author(s) 2021 